\title{
Normokalemic adenosine-lidocaine cardioplegia: Importance of maintaining a polarized myocardium for optimal arrest and reanimation
}

\author{
Kathryn L. Sloots, BSc (Hons), and Geoffrey P. Dobson, PhD
}

Objective: Depolarizing potassium cardioplegia does not afford optimal cardioprotection in pediatric or adult patients requiring complicated operative procedures. Polarizing adenosine-lidocaine cardioplegia has been shown to be cardioprotective without hyperkalemia. Our aim was to examine the effects of changing extracellular potassium levels in adenosine-lidocaine cardioplegia on arrest and reanimation properties.

Methods: Isolated-perfused rat hearts $(\mathrm{n}=96)$ were arrested at $32^{\circ} \mathrm{C}$ to $33^{\circ} \mathrm{C}$ for 1 or 2 hours with intermittent $200 \mu \mathrm{mol} / \mathrm{L}$ adenosine and $500 \mu \mathrm{mol} / \mathrm{L}$ lidocaine in modified Krebs-Henseleit buffer with $0.1,3.0,5.9,10$, and $16 \mathrm{mmol} / \mathrm{L}$ potassium or with 16 or $25 \mathrm{mmol} / \mathrm{L}$ potassium in Krebs-Henseleit buffer ( $\mathrm{n}=8$ for each group). Membrane potentials were estimated in the arrested ventricular myocardium $(n=42)$, and recovery function was measured in working mode during 60 minutes' reperfusion.

Results: Arrest was interrupted by breakout beats in the adenosine-lidocaine hypokalemic $(0.1$ and $3 \mathrm{mmol} / \mathrm{L}$ potassium) and non-adenosine-lidocaine hyperkalemic (16 and $25 \mathrm{mmol} / \mathrm{L}$ potassium) groups. The membrane potentials for the non-adenosine-lidocaine 16 and $25 \mathrm{mmol} / \mathrm{L}$ potassium groups were -51 and $-39 \mathrm{mV}$, and those for the adenosine-lidocaine groups $(0.1,3.0,5.9,10$, and $16 \mathrm{mmol} / \mathrm{L}$ potassium $)$ were $-183,-94,-75,-65$, and $-49 \mathrm{mV}$, respectively. After 1 hour of arrest, coronary vascular resistance increased linearly in adenosine-lidocaine cardioplegia with increasing potassium levels $(5.9,10$, and $16 \mathrm{mmol} / \mathrm{L})$, and the slope increased more than 2 -fold after 2 hours. Nearly $40 \%$ of hearts in the adenosine-lidocaine $(0.1 \mathrm{mmol} / \mathrm{L}$ potassium $)$ and nonadenosine-lidocaine $25 \mathrm{mmol} / \mathrm{L}$ potassium groups failed to recover after 1 hour arrest. After 2 hours, hearts in the polarizing $(5.9 \mathrm{mmol} / \mathrm{L}$ potassium $)$ adenosine-lidocaine group increased coronary vascular resistance by only $30 \%$ and spontaneously recovered $107 \%$ heart rate, $92 \%$ systolic pressure, $81 \%$ aortic flow, and $113 \%$ coronary flow (all metrics returned $85 \%$ to $100 \%$ at 15 minutes) with no reperfusion arrhythmias. In contrast, hearts in the adenosine-lidocaine $(3,10$, and $16 \mathrm{mmol} / \mathrm{L}$ potassium) groups were all slow to recover $(15 \%$ to $40 \%$ return at 15 minutes) and experienced arrhythmias. Increasing potassium levels in adenosine-lidocaine cardioplegia from 5.9 to $16 \mathrm{mmol} / \mathrm{L}$ resulted in a $67 \%$ loss of left ventricular contractility.

Conclusions: Polarizing adenosine-lidocaine cardioplegia $(5.9 \mathrm{mmol} / \mathrm{L}$ potassium $)$ administered intermittently at $33^{\circ} \mathrm{C}$ provides superior arrest and reanimation profiles under normokalemic conditions when the myocardial cell membrane potential is close to its resting state. (J Thorac Cardiovasc Surg 2010;139:1576-86)

Cardiac surgeons are currently facing unprecedented challenges and pressures from health care providers, surgeon shortages, and a sicker, older patient cohort with more diffuse multivessel disease and a higher incidence of diabetes, obesity, metabolic disorders, renal disease, failed angioplasty, and redo operations. ${ }^{1}$ In the new era of cardiac

From the Heart Research Laboratory, Department of Physiology and Pharmacology, Molecular Science Building, James Cook University, Townsville, Queensland, Australia.

Disclosures: G. P. Dobson reports lecture fees and equity in Hibernation Therapeutics. Supported in part by National Heart Foundation grant G 05B 2034 (to GPD) and National Health and Medical Research grant 540409 (to GPD).

Received for publication July 16, 2009; revisions received Sept 18, 2009; accepted for publication Oct 4, 2009; available ahead of print Feb 4, 2010.

Address for reprints: Geoffrey P. Dobson, PhD, Department of Physiology and Pharmacology, Molecular Science Building, James Cook University, Townsville, Queensland 4811, Australia (E-mail: geoffrey.dobson@jcu.edu.au).

0022-5223/\$36.00

Copyright (c) 2010 by The American Association for Thoracic Surgery doi: $10.1016 /$ j.jtcvs.2009.10.013 surgery, the older patient presenting with a diseased heart without complicating comorbidities will be a surgical rarity.

High-potassium cardioplegia has been identified as a major factor associated with poor outcomes in adult bypass, valvular, or pediatric congenital corrective operations. ${ }^{2,3}$ For more than 40 years, surgeons and scientists have sought alternatives to high-potassium cardioplegia, which arrests the heart at depolarized membrane potentials $(-50$ to $-60 \mathrm{mV}) \cdot{ }^{4-8}$ Potassium $\left(\mathrm{K}^{\dagger}\right)$-induced depolarization and the resulting intracellular $\mathrm{Na}^{+}$and $\mathrm{Ca}^{2+}$ loading can lead to myocardial and microvascular injury, ${ }^{9}$ coronary vasoconstriction and spasm, ${ }^{9}$ arrhythmias, ${ }^{10}$ and right and left ventricular stunning ${ }^{11}$ and potentiate the local inflammatory response with free radical production. ${ }^{5,11}$ Apart from electrochemical arrest, hyperkalemic cardioplegia offers the patient little or no cardioprotection.

For more than 10 years, our laboratory has developed a normokalemic nondepolarizing cardioplegia to place the heart in a more natural state of suspended animation and 


$$
\begin{aligned}
& \text { Abbreviations and Acronyms } \\
& \qquad \begin{aligned}
\mathrm{AF} & =\text { aortic flow } \\
\mathrm{AL} & =\text { adenosine-lidocaine } \\
\mathrm{CF} & =\text { coronary flow } \\
\mathrm{CO} & =\text { cardiac output } \\
\mathrm{CVR} & =\text { coronary vascular resistance } \\
\mathrm{HR} & =\text { heart rate } \\
\mathrm{K}^{+} & =\text {potassium } \\
\mathrm{K}-\mathrm{H} & =\text { Krebs-Henseleit } \\
\mathrm{SV} & =\text { stroke volume }
\end{aligned}
\end{aligned}
$$

arousal akin to natural hibernation. ${ }^{6}$ Adenosine and lidocaine, the active components of adenosine-lidocaine (AL) cardioplegia, clamp the membrane potential close to its resting voltage of $-83 \mathrm{mV}$, reduce coronary vascular resistance (CVR), and improve functional recovery. ${ }^{12} \mathrm{AL}$ cardioplegia can arrest the heart for up to 4 hours at $29^{\circ} \mathrm{C}$ with small increases in CVR, $85 \%$ to $100 \%$ recovery of heart rate (HR) and systolic pressure, and $70 \%$ to $80 \%$ recovery of cardiac output (CO), whereas only $17 \%$ of hearts arrested with hyperkalemic St Thomas Hospital solution no. 2 (Plegisol; Hospira, Inc, Lake Forest, Ill) survived. ${ }^{12}$ Further proof of concept was demonstrated by using AL all-blood cardioplegia in the canine model of cardiopulmonary bypass. ${ }^{13}$ More recently, we have shown that AL cardioplegia can be delivered continuously or intermittently at $33^{\circ} \mathrm{C}$ with no significant loss in functional parameters, ${ }^{7}$ and we are currently developing the concept for heart transplantation. ${ }^{8}$ At nonarrest concentrations, AL intravenous infusions during regional ischemia have also been shown to reduce mortality, arrhythmias, and infarct size ${ }^{14}$; however, there is much controversy about whether a lidocaine bolus administered during ischemia followed by adenosine at reperfusion has similar cardioprotective effects (Canyon and Dobson, unpublished data). ${ }^{15-17}$

The aim of the present study was to examine the effect of varying levels of extracellular $\mathrm{K}^{+}$in $\mathrm{AL}$ cardioplegia (0.1, 3.0, 5.9, 10 , and $16 \mathrm{mmol} / \mathrm{L} \mathrm{K}^{\dagger}$ ) on diastolic membrane potential, CVR, incidence of arrhythmias, time to first beat, $\mathrm{HR}$, systolic pressures, aortic and coronary flows (CFs), $\mathrm{CO}$, and stroke volume (SV) after 1 and 2 hours of arrest at $32^{\circ} \mathrm{C}$ to $33^{\circ} \mathrm{C}$. We report that polarized $\mathrm{AL}$ cardioplegia $\left(5.9 \mathrm{mmol} / \mathrm{L} \mathrm{K}^{+}\right)$provides superior arrest and recovery profiles and might offer an alternative to hyperkalemic solutions in pediatric and adult cardiac surgery.

\section{MATERIALS AND METHODS Animals}

Male Sprague-Dawley rats (390-450 g) were fed ad libitum and housed in a 14/10-hour light/dark cycle. Rats were anesthetized with an intraperitoneal injection of thiopentone sodium ( $100 \mathrm{mg} / \mathrm{kg}$ body weight $)$ and handled in compliance with James Cook University Ethics approval number A1107 and with the "Guide for care and use of laboratory animals" from the National Institutes of Health (publication no. 85-23, Public Health Service publication 1996). Adenosine and other chemicals were obtained from Sigma Chemical Co (Castle Hill, New South Wales, Australia). Lidocaine hydrochloride was purchased as a 2\% solution (ilium) from Lyppard (Queensland, Australia).

\section{Composition of Buffers and Arrest Solutions}

Krebs-Henseleit perfusion buffer. Hearts were perfused in the Langendorff and working mode with a modified Krebs-Henseleit $(\mathrm{K}-\mathrm{H})$ buffer containing $10 \mathrm{mmol} / \mathrm{L}$ glucose $(117 \mathrm{mmol} / \mathrm{L} \mathrm{NaCl}, 5.9 \mathrm{mmol} / \mathrm{L}$ $\mathrm{KCl}, 25 \mathrm{mmol} / \mathrm{L} \mathrm{NaHCO}_{3}, 1.2 \mathrm{mmol} / \mathrm{L} \mathrm{NaH}_{2} \mathrm{PO}_{4}, 1.12 \mathrm{mmol} / \mathrm{L} \mathrm{CaCl}_{2}$ [free $\mathrm{Ca}^{2+}=1.07 \mathrm{mmol} / \mathrm{L}$ ], and $0.512 \mathrm{mmol} / \mathrm{L} \mathrm{MgCl}_{2}\left[\right.$ free $\mathrm{Mg}^{2+}=0.5$ $\mathrm{mmol} / \mathrm{L}], \mathrm{pH} 7.4$ ) and bubbled with $95 \% \mathrm{O}_{2} / 5 \% \mathrm{CO}_{2}$ at $37^{\circ} \mathrm{C}^{7,12}$

AL arrest solutions. Adenosine $(200 \mu \mathrm{mol} / \mathrm{L})$ and lidocaine $(500$ $\mu \mathrm{mol} / \mathrm{L}$ ) were added to $\mathrm{K}-\mathrm{H}$ buffer containing either $0.1,3,5.9,10$, or 16 $\mathrm{mmol} / \mathrm{L} \mathrm{K}^{+}$with $10 \mathrm{mmol} / \mathrm{L}$ glucose, $\mathrm{pH}$ 7.7. .,12 $^{-12}$

Hyperkalemic arrest solutions. $\mathrm{K}^{+}, 16$ and $25 \mathrm{mmol} / \mathrm{L}$, was added to $\mathrm{K}-\mathrm{H}$ buffer containing $10 \mathrm{mmol} / \mathrm{L}$ glucose, filtered, and maintained at $37^{\circ} \mathrm{C}$. The total $\mathrm{K}^{+}$concentration in these solutions was 16 and $25 \mathrm{mmol} / \mathrm{L}$ (as $\mathrm{KCl}$ ). Arrest solutions were filtered with a $0.2-\mu \mathrm{m}$ filter and not bubbled.

\section{Experimental Protocols and Groups}

Rats were randomly assigned to one of 3 experiments and 20 groups. Cardiac membrane potential. There were 8 groups $(n=6$ for each group), control (nonarrest) and immediately after arrest (at induction): AL $0.1,3,5.9,10$, and $16 \mathrm{mmol} / \mathrm{L} \mathrm{K}^{+}$and 16 and $25 \mathrm{mmol} / \mathrm{L} \mathrm{K}^{+}$alone.

One-hour arrest. There were 7 groups ( $\mathrm{n}=8$ for each group): $\mathrm{AL}$ $0.1,3,5.9,10$, and $16 \mathrm{mmol} / \mathrm{L} \mathrm{K}^{+}$and 16 and $25 \mathrm{mmol} / \mathrm{L} \mathrm{K}^{+}$alone.

Two-hour arrest. There were 5 groups ( $\mathrm{n}=8$ for each group): AL 3 , $5.9,10$, and $16 \mathrm{mmol} / \mathrm{L} \mathrm{K}^{+}$and $16 \mathrm{mmol} / \mathrm{L} \mathrm{K}^{+}$alone. Note that we did not include $\mathrm{AL} 0.1 \mathrm{mmol} / \mathrm{L} \mathrm{K}^{+}$or $25 \mathrm{mmol} / \mathrm{L} \mathrm{K}^{+}$alone groups because $40 \%$ of them failed to recover after 1 hour's arrest.

\section{Langendorff and Working Rat Heart Preparation}

Hearts were placed in ice-cold $\mathrm{K}-\mathrm{H}$ buffer, cleaned, and then connected through the aorta to a standard Langendorff apparatus and perfused in a retrograde fashion at $80 \mathrm{~cm} \mathrm{H}_{2} \mathrm{O}(60 \mathrm{~mm} \mathrm{Hg}){ }^{12}$ The pulmonary artery and left atrium were cannulated, and the heart was switched to working mode. The preload was $10 \mathrm{~cm} \mathrm{H}_{2} \mathrm{O}$, and the afterload was $100 \mathrm{~cm} \mathrm{H}_{2} \mathrm{O}(76 \mathrm{~mm} \mathrm{Hg})$. After 10 minutes of stabilization, hearts were switched to nonworking mode to administer the arrest solution. $\mathrm{HR}$, aortic pressure, $\mathrm{CF}$, and aortic flow $\mathrm{AF}$ ) were measured before, during, and after arrest, as reported previously. ${ }^{7,12}$ After arrest, hearts were switched to working mode, and function was monitored, as described previously. ${ }^{7,12}$ No pacing or cardiac massage was used. The heart's temperature was measured with a Cole-Palmer temperature sensor (8402-20; Cole-Parmer, Vernon Hills, Ill) tucked under the left auricle. ${ }^{7}$ Breakout beats were monitored visually, timed, and recorded during induction of arrest and during and between maintenance cardioplegic flushes. Heart rhythm was not assessed with an electrocardiogram, but irregularities in rhythm were noted, together with their effect on HR, developed pressures, and CO.

\section{Arrest Induction Protocol}

Fifty milliliters of cardioplegia was administered at $60 \mathrm{~mm} \mathrm{Hg}$, and the aorta was crossclamped for 18 minutes, after which a 2-minute infusion pulse of cardioplegia was administered and the clamp was reapplied. Cardioplegic volumes were measured for estimating CVR. ${ }^{7}$ The arrest temperature of $32^{\circ} \mathrm{C}$ to $33^{\circ} \mathrm{C}$ was chosen from the studies that reported that tepid temperatures provided optimal heart recovery and brain protection during cardiac surgery. ${ }^{7}$ Estimation of myocardial membrane potential and CVR can be found in Appendix 1. 


\section{Statistical Analysis}

All results are expressed as means \pm standard errors of the mean. Data from the 1- and 2-hour protocols were analyzed separately. One-way analysis of variance was used to compare data at specific time points (eg, arrest time and time to first reperfusion beat; Tables 1 and 2 and Figures $1, B ; 2, A$ and $B$; and 3,A). Two-way analysis of variance with repeated measures was used to compare functional parameters of the different cardioplegia groups across multiple time points during the arrest period and 60 minutes of reperfusion (Figures 1, $B$, and 3, $A$ and $B$ ). Significance was assessed with Tukey and Dunnett (2-sided) post-hoc tests. The alpha level of significance for all experiments was set at $P<.05$.

\section{RESULTS}

\section{Arrest Times and Membrane Potentials}

Times to arrest for the AL cardioplegia groups were not significantly different and ranged from $7.4 \pm 0.6$ to $11 \pm$ 1.3 seconds. Arrest times were significantly increased in hearts perfused with $16 \mathrm{mmol} / \mathrm{L} \mathrm{K}^{+}$alone $(22 \pm 2$ to $28 \pm$ 8 seconds) and decreased in the presence of $25 \mathrm{mmol} / \mathrm{L} \mathrm{K}^{+}$ alone (13.4 \pm 1.1 seconds). Nearly all hearts perfused with hypokalemic $\mathrm{AL}$ solutions $\left(0.1\right.$ and $\left.3 \mathrm{mmol} / \mathrm{L} \mathrm{K}^{+}\right)$ failed to maintain arrest; breakout beats occurred for $9.8 \pm$ 1.3 minutes and $7.1 \pm 0.6$ minutes, respectively. Similarly, hearts perfused with $16 \mathrm{mmol} / \mathrm{L} \mathrm{K}^{+}$alone and $25 \mathrm{mmol} / \mathrm{L}$ $\mathrm{K}^{+}$alone had breakout beats lasting for 2.5 to 25 minutes and for 8 seconds to 11 minutes, respectively, after which arrest occurred. The membrane potential for each group is found in Figure 1, $A$, and Table 1. The prearrest value was
$-78 \pm 1 \mathrm{mV}$ at $37^{\circ} \mathrm{C}$. Total tissue water content was not significantly different among the groups $(86.0 \pm 0.4 \%$ to 87.1 $\pm 0.5 \%, \mathrm{n}=6$ each).

\section{CVR During Induction and 2-Minute "Flushes"}

During induction, there were no significant differences in the rate of coronary outflow in the AL groups, although a $5 \%$ increase occurred in the $\mathrm{AL} 5.9 \mathrm{mmol} / \mathrm{L} \mathrm{K}^{+}$group. In contrast, the rates in the $16 \mathrm{mmol} / \mathrm{L}$ and $25 \mathrm{mmol} / \mathrm{L} \mathrm{K}^{+}$ alone groups were significantly decreased $(24 \%$ and $35 \%$ decrease, respectively; Table 1). The significant increase in CVR for the $16 \mathrm{mmol} / \mathrm{L} \mathrm{K}^{+}$group is also shown on the $\mathrm{y}$-axis of Figure 1, $B$ ("end of induction").

Maintenance cardioplegic volumes and CVR measured during 2-minute flushes every 18 minutes over 1 and 2 hours' arrest are shown in Table 1 and Figure 1, B. Significantly greater volumes were delivered to the AL 5.9 $\mathrm{mmol} / \mathrm{L} \mathrm{K}^{+}$and $\mathrm{AL} 10 \mathrm{mmol} / \mathrm{L} \mathrm{K}^{+}$groups. After 1 hour's arrest, the $\mathrm{AL} 0.1 \mathrm{mmol} / \mathrm{L} \mathrm{K}^{+}$and $25 \mathrm{mmol} / \mathrm{L} \mathrm{K}^{+}$alone groups had the highest CVRs. Nearly $40 \%$ (3/8) of hearts in each of these 2 groups subsequently failed to recover aortic/coronary flows after 1 hour's arrest, and therefore we did not include them in the longer arrest study. After 2 hours' arrest, the AL $5.9 \mathrm{mmol} / \mathrm{L} \mathrm{K}^{+}$group had the lowest CVR (Figure 1,B). As the membrane potential deviated from near-resting voltage (ie, from AL $5.9 \mathrm{mmol} / \mathrm{L}$

TABLE 1. Arrest parameters: 1 - and 2-hour warm arrest protocols $\left(32^{\circ} \mathrm{C}-33^{\circ} \mathrm{C}\right)$

\begin{tabular}{|c|c|c|c|c|c|c|c|}
\hline \multirow[b]{3}{*}{ Arrest solution } & \multirow{3}{*}{$\begin{array}{c}\text { Diastolic } \\
\text { membrane } \\
\text { potential }(\mathbf{n}=\mathbf{6})\end{array}$} & \multicolumn{3}{|c|}{ 1-h Arrest } & \multicolumn{3}{|c|}{ 2-h Arrest } \\
\hline & & \multirow{2}{*}{$\begin{array}{c}\text { Cardioplegic } \\
\text { flush volume } \\
\qquad(\mathrm{mL})^{*}\end{array}$} & \multicolumn{2}{|c|}{$\begin{array}{c}\text { Coronary } \\
\text { vascular resistance } \\
\left(\text { megadyne } \cdot \mathbf{s}^{-1} \cdot \mathbf{c m}^{-5}\right)\end{array}$} & \multirow{2}{*}{$\begin{array}{l}\text { Cardioplegic } \\
\text { flush volume } \\
(\mathrm{mL})^{*}\end{array}$} & \multicolumn{2}{|c|}{$\begin{array}{c}\text { Coronary } \\
\text { vascular resistance } \\
\left(\text { megadyne } \cdot \mathbf{s}^{-1} \cdot \mathbf{c m}^{-5}\right)\end{array}$} \\
\hline & & & End induction & At 58 min arrest & & End induction & At $118 \mathrm{~min}$ arrest \\
\hline $\mathrm{AL}\left(0.1 \mathrm{mmol} / \mathrm{L} \mathrm{K}^{+}\right)$ & $-183 \pm 1 \mathrm{mV}$ & $74 \pm 2$ & $0.25 \pm 0$ & $0.53 \pm .02 \#$ & ND & ND & ND \\
\hline $\mathrm{AL}\left(3.0 \mathrm{mmol} / \mathrm{L} \mathrm{K}^{+}\right)$ & $-94 \pm 1 \mathrm{mV}$ & $87 \pm 3$ & $0.26 \pm 0.01$ & $0.40 \pm 0.03$ & $138 \pm 5$ & $0.25 \pm 0.01$ & $0.65 \pm 0.06$ \\
\hline $\mathrm{AL}\left(5.9 \mathrm{mmol} / \mathrm{L} \mathrm{K}^{+}\right)$ & $-75 \pm 2 \mathrm{mV}$ & $104 \pm 1 \dagger$ & $0.24 \pm 0.01$ & $0.29 \pm 0.01$ & $203 \pm 3 \ddagger \ddagger$ & $0.24 \pm 0.01$ & $0.31 \pm 0.01\|\|$ \\
\hline $\mathrm{AL}\left(10 \mathrm{mmol} / \mathrm{L} \mathrm{K}^{+}\right)$ & $-65 \pm 1 \mathrm{mV}$ & $109 \pm 3 \ddagger$ & $0.24 \pm 0.01$ & $0.28 \pm 0.02$ & $155 \pm 4$ & $0.27 \pm 0.01$ & $0.47 \pm 0.03$ \\
\hline $\mathrm{AL}\left(16 \mathrm{mmol} / \mathrm{L} \mathrm{K}^{+}\right)$ & $-49 \pm 1 \mathrm{mV}$ & $89 \pm 3$ & $0.26 \pm 0.01$ & $0.40 \pm 0.06$ & $157 \pm 6$ & $0.28 \pm 0.01$ & $0.57 \pm 0.07$ \\
\hline $16 \mathrm{mmol} / \mathrm{L} \mathrm{K}^{+}$ & $-51 \pm 1 \mathrm{mV}$ & $71 \pm 2$ & $0.35 \pm 0.02 \|$ & $0.46 \pm 0.03^{* *}$ & $139 \pm 5$ & $0.40 \pm 0.03 \S \S$ & $0.57 \pm 0.05$ \\
\hline $25 \mathrm{mmol} / \mathrm{L} \mathrm{K}^{+}$ & $-39 \pm 1 \mathrm{mV}$ & $56 \pm 2 \S$ & $0.38 \pm 0.029$ & $0.61 \pm 0.05 \dagger \dagger$ & ND & ND & ND \\
\hline
\end{tabular}

The diastolic membrane potential of the left ventricle was calculated from the Nernstian distribution of potassium ions (see the Materials and Methods section). $A L$, Adenosinelidocaine; $N D$, not determined), AL $0.1 \mathrm{mmol} / \mathrm{L}$ potassium $\left(\mathrm{K}^{+}\right)$or $25 \mathrm{mmol} / \mathrm{L} \mathrm{K}^{+}$alone were not determined because approximately $40 \%$ failed to recover function after 1 hour arrest.

*Volume of cardioplegic solution administered during 2-minute flushes during the maintenance period.

$\dagger P<.01$ compared with AL $0.1 \mathrm{mmol} / \mathrm{L} \mathrm{K}^{+}$and $16 \mathrm{mmol} / \mathrm{L} \mathrm{K} \mathrm{K}^{+}$groups.

$\ddagger P<.01$ compared with AL $0.1 \mathrm{mmol} / \mathrm{L} \mathrm{K}^{+}$and $16 \mathrm{mmol} / \mathrm{L} \mathrm{K}$ groups, $P<.05$ compared with AL 3 and $16 \mathrm{mmol} / \mathrm{L} \mathrm{K}{ }^{+}$groups.

${ }_{\S} P<.01$ compared with AL $3,5.9,10$, and $16 \mathrm{mmol} / \mathrm{L} \mathrm{K}^{+}$and $16 \mathrm{mmol} / \mathrm{L} \mathrm{K} \mathrm{K}^{+}$groups, $P<.05$ compared with the $\mathrm{AL} 0.1 \mathrm{mmol} / \mathrm{L} \mathrm{K}^{+}$group.

$\| P<.01$ compared with all groups except $25 \mathrm{mmol} / \mathrm{L} \mathrm{K}^{+}$.

\ $P<.01$ compared with all groups except $16 \mathrm{mmol} / \mathrm{L} \mathrm{K}^{+}$.

$\# P<.05$ compared with AL 5.9 and $10 \mathrm{mmol} / \mathrm{L} \mathrm{K}^{+}$and $25 \mathrm{mmol} / \mathrm{L} \mathrm{K}^{+}$groups.

**P $<.01$ compared with AL 5.9 and $10 \mathrm{mmol} / \mathrm{L} \mathrm{K}^{+}$groups.

$\dagger \dagger P<.05$ compared with all other groups.

$\ddagger \ddagger P<.05$ compared with AL $3 \mathrm{mmol} / \mathrm{L} \mathrm{K}^{+}$and $16 \mathrm{mmol} / \mathrm{L} \mathrm{K} \mathrm{K}^{+}$groups.

$\S \S P<.01$ compared with all other groups.

\|\|$P<.01$ compared with AL 3 and $16 \mathrm{mmol} / \mathrm{L} \mathrm{K}^{+}$and $16 \mathrm{mmol} / \mathrm{L} \mathrm{K}^{+}$groups. 
TABLE 2. Functional parameters of isolated rat hearts before arrest and at 60 minutes' reperfusion (working mode) after 1 or 2 hours' arrest at $32^{\circ} \mathrm{C}$ to $33^{\circ} \mathrm{C}$

\begin{tabular}{|c|c|c|c|c|c|c|c|c|c|c|c|c|c|c|}
\hline \multirow{2}{*}{$\begin{array}{l}\text { Treatment groups } \\
(\mathrm{n}=\mathbf{8} \text { each group) }\end{array}$} & \multicolumn{2}{|c|}{ Heart rate } & \multicolumn{2}{|c|}{$\begin{array}{l}\text { Systolic } \\
\text { pressure }\end{array}$} & \multicolumn{2}{|c|}{$\begin{array}{l}\text { Diastolic } \\
\text { pressure }\end{array}$} & \multicolumn{2}{|c|}{ Aortic flow } & \multicolumn{2}{|c|}{ Coronary flow } & \multicolumn{2}{|c|}{$\begin{array}{c}\text { Cardiac } \\
\text { output }\end{array}$} & \multicolumn{2}{|c|}{ Stroke volume } \\
\hline & beats/min & $\% \mathbf{P A}$ & $\mathrm{mm} \mathrm{Hg}$ & $\% \mathbf{P A}$ & $\mathbf{m m ~ H g}$ & $\% \mathbf{P A}$ & $\mathrm{mL} / \mathrm{min}$ & $\% \mathbf{P A}$ & $\mathrm{mL} / \mathrm{min}$ & $\% \mathbf{P A}$ & $\mathrm{mL} / \mathrm{min}$ & $\% \mathbf{P A}$ & mL/beat & $\% \mathbf{P A}$ \\
\hline \multicolumn{15}{|l|}{$\mathrm{AL}\left(0.1 \mathrm{mmol} / \mathrm{L} \mathrm{K}^{\dagger}\right)$} \\
\hline Before arrest & $338 \pm 6$ & 61 & $133 \pm 2$ & 54 & $75 \pm 3$ & 66 & $56 \pm 2$ & 28 & $22 \pm 1$ & 41 & $78 \pm 2$ & 32 & $0.23 \pm 0.1$ & 32 \\
\hline 1-h Arrest & $206 \pm 61$ & & $71 \pm 21$ & & $51 \pm 15$ & & $16 \pm 69$ & & $9 \pm 3 \dagger$ & & $25 \pm 9$ & & $0.07 \pm 0.02$ & \\
\hline \multicolumn{15}{|l|}{$\mathrm{AL}\left(3 \mathrm{mmol} / \mathrm{L} \mathrm{K}^{\dagger}\right)$} \\
\hline Before arrest & $336 \pm 15$ & 99 & $129 \pm 4$ & 92 & $71 \pm 3$ & 105 & $57 \pm 2$ & 81 & $20 \pm 1$ & 90 & $77 \pm 2$ & 83 & $0.23 \pm 0.1$ & 84 \\
\hline 1-h Arrest & $329 \pm 7$ & & $118 \pm 3$ & & $74 \pm 2$ & & $46 \pm 2$ & & $18 \pm 1$ & & $64 \pm 3$ & & $0.19 \pm 0.01$ & \\
\hline Before arrest & $304 \pm 10$ & 45 & $131 \pm 3$ & 23 & $61 \pm 1$ & 32 & $60 \pm 2$ & 11 & $20 \pm 1$ & 17 & $79 \pm 3$ & 13 & $0.26 \pm 0.1$ & 13 \\
\hline 2-h arrest & $133 \pm 43^{*}$ & & $32 \pm 18^{*}$ & & $20 \pm 10^{*}$ & & $6 \pm 5 \|$ & & $4 \pm 2 \|$ & & $10 \pm 7 \|$ & & $0.04 \pm 0.02 \|$ & \\
\hline \multicolumn{15}{|c|}{$\mathrm{AL}\left(5.9 \mathrm{mmol} / \mathrm{L} \mathrm{K}^{\dagger}\right)$} \\
\hline Before arrest & $301 \pm 19$ & 110 & $131 \pm 4$ & 94 & $73 \pm 2$ & 100 & $56 \pm 2$ & 91 & $19 \pm 1$ & 101 & $75 \pm 3$ & 93 & $0.25 \pm 0.1$ & 86 \\
\hline 1-h Arrest & $327 \pm 16$ & & $124 \pm 4$ & & $73 \pm 2$ & & $51 \pm 3$ & & $19 \pm 1$ & & $70 \pm 3$ & & $0.22 \pm 0.01$ & \\
\hline Before arrest & $291 \pm 11$ & 107 & $130 \pm 1$ & 92 & $61 \pm 1$ & 100 & $59 \pm 2$ & 81 & $18 \pm 1$ & 113 & $77 \pm 2$ & 89 & $0.26 \pm 0.1$ & 84 \\
\hline 2-h Arrest & $309 \pm 9$ & & $120 \pm 2$ & & $61 \pm 1$ & & $48 \pm 2 \#$ & & $21 \pm 1 \#$ & & $69 \pm 2 \#$ & & $0.22 \pm 0.01 \#$ & \\
\hline \multicolumn{15}{|l|}{$\mathrm{AL}\left(10 \mathrm{mmol} / \mathrm{L} \mathrm{K}^{+}\right)$} \\
\hline Before arrest & $320 \pm 10$ & 102 & $132 \pm 2$ & 94 & $71 \pm 2$ & 110 & $60 \pm 1$ & 79 & $20 \pm 1$ & 97 & $79 \pm 1$ & 84 & $0.25 \pm 0.1$ & 83 \\
\hline 1 -h arrest & $325 \pm 9$ & & $124 \pm 2$ & & $78 \pm 3$ & & $47 \pm 1$ & & $19 \pm 1$ & & $66 \pm 2$ & & $0.21 \pm 0.01$ & \\
\hline Before arrest & $338 \pm 15$ & 95 & $124 \pm 1$ & 92 & $60 \pm 1$ & 107 & $56 \pm 1$ & 71 & $19 \pm 1$ & 81 & $75 \pm 2$ & 74 & $0.23 \pm 0.1$ & 80 \\
\hline 2-h arrest & $317 \pm 16$ & & $114 \pm 2$ & & $64 \pm 1$ & & $40 \pm 2$ & & $16 \pm 1$ & & $56 \pm 1$ & & $0.18 \pm 0.01$ & \\
\hline \multicolumn{15}{|l|}{$\mathrm{AL}\left(16 \mathrm{mmol} / \mathrm{L} \mathrm{K}^{+}\right)$} \\
\hline Before arrest & $309 \pm 15$ & 103 & $131 \pm 2$ & 93 & $71 \pm 2$ & 101 & $56 \pm 1$ & 81 & $19 \pm 1$ & 94 & $75 \pm 1$ & 84 & $0.25 \pm 0.1$ & 82 \\
\hline 1-h arrest & $318 \pm 15$ & & $122 \pm 3$ & & $72 \pm 3$ & & $45 \pm 2$ & & $18 \pm 2$ & & $18 \pm 2$ & & $0.20 \pm 0.01$ & \\
\hline Before arrest & $309 \pm 14$ & 64 & $139 \pm 2$ & 53 & $61 \pm 1$ & 65 & $57 \pm 2$ & 29 & $19 \pm 1$ & 33 & $76 \pm 2$ & 36 & $0.25 \pm 0.1$ & 39 \\
\hline 2-h Arrest & $208 \pm 33$ & & $74 \pm 23$ & & $39 \pm 11$ & & $20 \pm 8$ & & $8 \pm 3$ & & $28 \pm 11$ & & $0.10 \pm 0.04$ & \\
\hline \multicolumn{15}{|l|}{$16 \mathrm{mmol} / \mathrm{L} \mathrm{K}^{+}$alone } \\
\hline Before arrest & $329 \pm 6$ & & $133 \pm 2$ & & $73 \pm 3$ & & $59 \pm 1$ & & $19 \pm 1$ & & $78 \pm 2$ & & $0.24 \pm 0.1$ & \\
\hline 1-h arrest & $306 \pm 25$ & 94 & $117 \pm 3$ & 88 & $78 \pm 3$ & 107 & $40 \pm 5$ & 69 & $15 \pm 1$ & 81 & $55 \pm 5$ & 72 & $0.18 \pm 0.01$ & 76 \\
\hline Before arrest & $304 \pm 9$ & & $134 \pm 3$ & & $61 \pm 1$ & & $56 \pm 1$ & & $18 \pm 1$ & & $75 \pm 2$ & & $0.25 \pm 0.1$ & \\
\hline 2-h arrest & $248 \pm 47$ & 76 & $74 \pm 21$ & 56 & $41 \pm 12$ & 65 & $25 \pm 8$ & 40 & $10 \pm 3$ & 49 & $35 \pm 10$ & 46 & $0.11 \pm 0.03$ & 43 \\
\hline \multicolumn{15}{|l|}{$25 \mathrm{mmol} / \mathrm{L} \mathrm{K}^{+}$alone } \\
\hline Before arrest & $306 \pm 12$ & & $136 \pm 3$ & & $66 \pm 3$ & & $59 \pm 1$ & & $20 \pm 1$ & & $79 \pm 1$ & & $0.26 \pm 0.1$ & \\
\hline 1-h arrest & $189 \pm 56 \dagger$ & 63 & $78 \pm 23 \ddagger$ & 57 & $44 \pm 13 \S$ & 66 & $26 \pm 8 \dagger$ & 43 & $11 \pm 3 \dagger$ & 54 & $37 \pm 11 * *$ & 46 & $0.12 \pm 0.04 \dagger$ & 46 \\
\hline
\end{tabular}

$\% P A$, Percentage of prearrest value; $A L$, adenosine-lidocaine.

$* P<.01$ compared with AL 5.9 and $10 \mathrm{mmol} / \mathrm{L}$ potassium $(\mathrm{K}+)$ groups.

$\dagger P<.05$ compared with $\mathrm{AL} 3,5.9,10$, and $16 \mathrm{mmol} / \mathrm{L} \mathrm{K} \mathrm{K}^{+}$groups.

$\ddagger P<.05$ compared with AL 5.9 and $10 \mathrm{mmol} / \mathrm{L} \mathrm{K}^{+}$groups.

$\S P<.05$ compared with $\mathrm{AL} 3$ and $10 \mathrm{mmol} / \mathrm{L} \mathrm{K}^{+}$and $16 \mathrm{mmol} / \mathrm{L} \mathrm{K}^{+}$alone groups.

$\| P<.01$ compared with the $\mathrm{AL} 10 \mathrm{mmol} / \mathrm{L} \mathrm{K}^{+}$group.

$\mathbb{\Upsilon} P<.01$ compared with all groups except the $25 \mathrm{mmol} / \mathrm{L} \mathrm{K}{ }^{+}$alone group.

$\# P<.01$ compared with the AL 3 and $16 \mathrm{mmol} / \mathrm{L} \mathrm{K}^{+}$and $16 \mathrm{mmol} / \mathrm{L} \mathrm{K}^{+}$alone groups.

$* * P<.01$ compared with the $\mathrm{AL} 3,5.9,10$, and $16 \mathrm{mmol} / \mathrm{L} \mathrm{K} \mathrm{K}^{+}$groups.

$\mathrm{K}^{+}$), the CVR increased, and this effect was magnified as arrest time increased (Figure 1, C).

\section{Time to First Spontaneous Beat and Aortic Flow}

After 1 hour's arrest, times to first beat increased from 0.9 minutes (AL $0.1 \mathrm{mmol} / \mathrm{L} \mathrm{K}^{+}$) to 5.2 minutes $(25$ $\mathrm{mmol} / \mathrm{L} \mathrm{K}^{+}$alone; Figure 2, A). The effect of doubling crossclamp time led to longer times to first beat, with the exception of the $\mathrm{AL} 5.9 \mathrm{mmol} / \mathrm{L} \mathrm{K} \mathrm{K}^{+}$and $\mathrm{AL} 3.0$ $\mathrm{mmol} / \mathrm{L} \mathrm{K}^{+}$groups (Figure 2, A). Time to first $\mathrm{AF}$ in- creased from 3.1 minutes $\left(16 \mathrm{mmol} / \mathrm{L} \mathrm{K}^{+}\right.$alone) to 13.9 minutes (AL $16 \mathrm{mmol} / \mathrm{L} \mathrm{K}^{+}$; Figure 2, B). Doubling arrest time resulted in up to 2.4 longer times to generate $\mathrm{AF}$ (Figure $2, B$ ). Nineteen hearts failed to achieve AF in our study: AL $0.1 \mathrm{mmol} / \mathrm{L} \mathrm{K}^{+}$(3 hearts after 1 hour's arrest), AL $3 \mathrm{mmol} / \mathrm{L} \mathrm{K}^{+}$(6 hearts after 2 hours' arrest), AL 16 $\mathrm{mmol} / \mathrm{L} \mathrm{K}^{+}$(4 hearts after 2 hours' arrest), $16 \mathrm{mmol} / \mathrm{L}$ $\mathrm{K}^{+}$alone ( 3 hearts after 2 hours' arrest), and $25 \mathrm{mmol} / \mathrm{L}$ $\mathrm{K}^{+}$alone (3 hearts after 1 hour's arrest). AL $5.9 \mathrm{mmol} / \mathrm{L}$ $\mathrm{K}^{+}$and $\mathrm{AL} 10 \mathrm{mmol} / \mathrm{L} \mathrm{K}^{+}$were the only 2 groups in which 


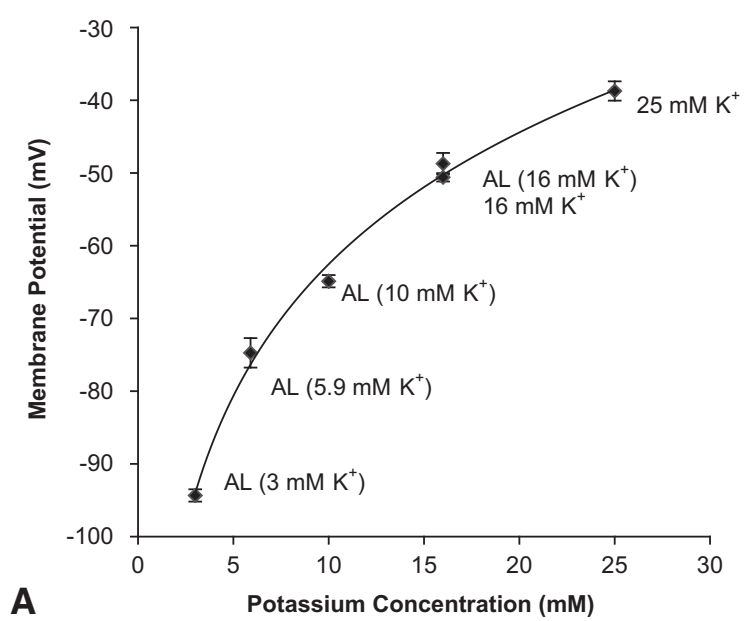

A
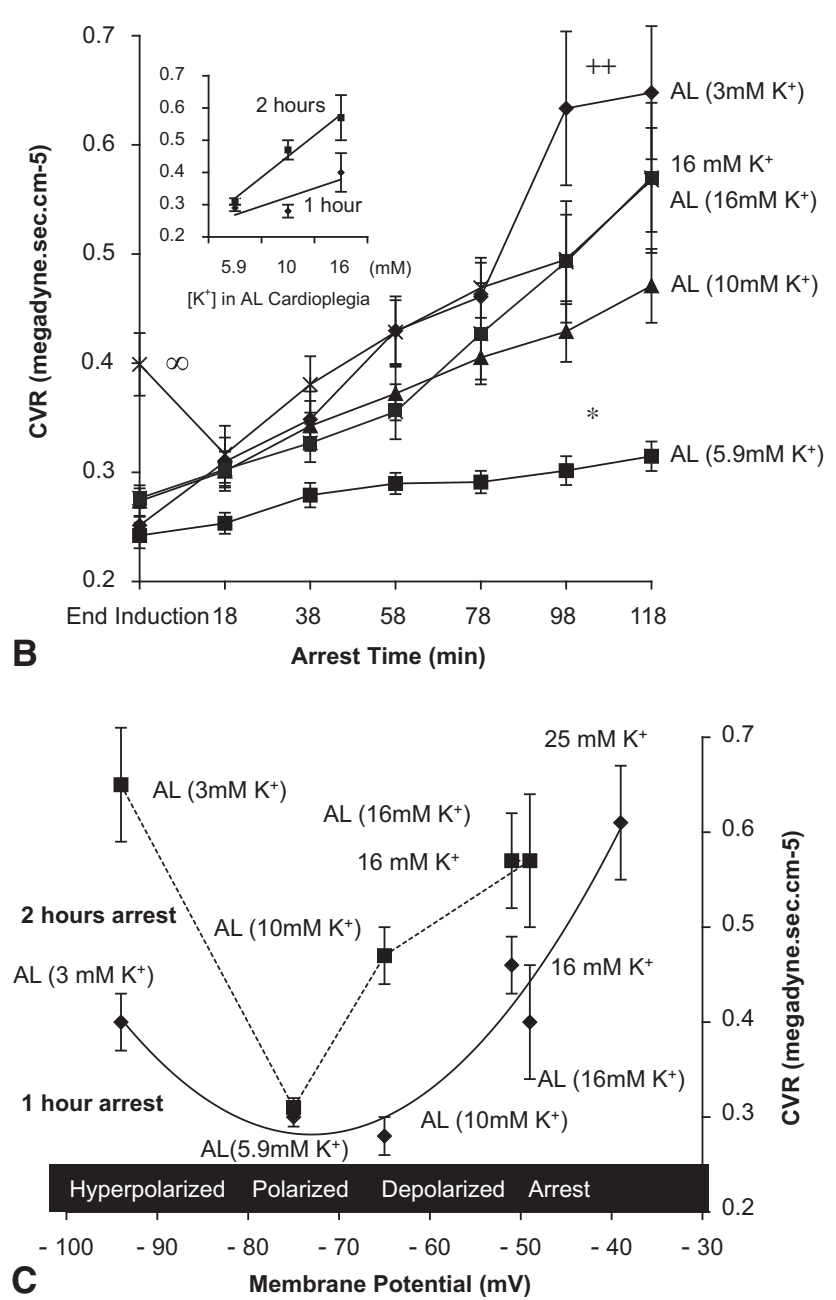

FIGURE 1. A, The effect of increasing potassium $\left(\mathrm{K}^{\dagger}\right)$ concentrations on the left ventricular diastolic membrane potential in the isolated Langendorff rat heart arrested with adenosine-lidocaine $(A L)$ cardioplegia (3.0, 5.9, 10, and $16 \mathrm{mmol} / \mathrm{L} \mathrm{K}^{+}$) or $16 \mathrm{mmol} / \mathrm{L} \mathrm{K}^{+}$alone and $25 \mathrm{mmol} / \mathrm{L} \mathrm{K}^{+}$alone in Krebs-Henseleit buffer. Membrane potentials $(\varphi)$ were calculated from the Nerstian distributions of $\mathrm{K}^{+}$(see the Materials and Methods section for details). The relationship between diastolic membrane potential and extracellular $\mathrm{K}^{+}$is as follows: $\varphi(\mathrm{mV})=26.23 \ln \left[\mathrm{K}^{+}\right]-123.44\left(\mathrm{R}^{2}=0.99\right)$, all hearts recovered AF after 1 and 2 hours' arrest at $33^{\circ} \mathrm{C}$. The effect of membrane potential and increasing $\mathrm{K}^{+}$concentrations in AL cardioplegia on time to first beat and $\mathrm{AF}$ are shown in Figures 2, $C$ and $D$.

\section{Early Reperfusion Ventricular Arrhythmias}

During reanimation and before AF was achieved, abnormal rhythms were observed in hearts from the AL 0.1 $\mathrm{mmol} / \mathrm{L} \mathrm{K}^{+}$(2 hearts), $16 \mathrm{mmol} / \mathrm{L} \mathrm{K}^{+}$alone (1 heart), and $25 \mathrm{mmol} / \mathrm{L} \mathrm{K}^{+}$alone (2 hearts) groups arrested for 1 hour, and after 2 hours in the $\mathrm{AL} 10 \mathrm{mmol} / \mathrm{L} \mathrm{K}^{+}$(3 hearts), $\mathrm{AL}$ $16 \mathrm{mmol} / \mathrm{L} \mathrm{K}^{+}$(4 hearts), and $16 \mathrm{mmol} / \mathrm{L} \mathrm{K}^{+}$alone (2 hearts) groups.

\section{Functional Recovery}

Recovery of HR, systolic and diastolic pressures, aortic and coronary flows, $\mathrm{CO}$, and SV for the 1- and 2-hour arrest groups are shown in Table 2 and Figure 3. During prearrest, there were no significant differences between the groups. After 1 and 2 hours' arrest, the $\mathrm{AL} 5.9 \mathrm{mmol} / \mathrm{L} \mathrm{K}^{+}$group recovered $107 \%$ to $110 \%$ of its prearrest HR at 60 minutes' reperfusion (94\% to $98 \%$ of recovery occurred at $15 \mathrm{~min}$ utes), which was significantly higher than that seen in the

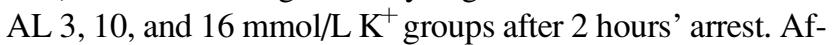
ter 1 hour's arrest, the $\mathrm{AL} 10,16$, and $3 \mathrm{mmol} / \mathrm{L} \mathrm{K}^{+}$and 16 $\mathrm{mmol} / \mathrm{L} \mathrm{K}^{+}$alone groups recovered $84 \%, 70 \%, 79 \%$, and $99 \%$ of their prearrest $\mathrm{HR}$ at 15 minutes' and $102 \%$, $103 \%, 99 \%$, and $94 \%$ at 60 minutes' reperfusion, respectively. After 2 hours' arrest, the AL $10 \mathrm{mmol} / \mathrm{L} \mathrm{K}^{+}$group recovered $95 \%$ at 60 minutes; however, only $36 \%$ recovery occurred at 15 minutes. The $16 \mathrm{mmol} / \mathrm{L} \mathrm{K}^{+}$alone, AL 16 $\mathrm{mmol} / \mathrm{L} \mathrm{K}^{+}$, and $\mathrm{AL} 3 \mathrm{mmol} / \mathrm{L} \mathrm{K}^{+}$groups recovered $79 \%$, $28 \%$, and $39 \%$ of their respective pre-arrest HRs at 15 minutes' and $76 \%, 64 \%$, and $45 \%$ at 60 minutes' reperfusion (Table 2). The relationship between HR and increasing extracellular $\mathrm{K}^{+}$concentrations in AL cardioplegia at 15 minutes' reperfusion after 1 hour's arrest was as follows: HR

where $\left[\mathrm{K}^{+}\right]$is the extracellular $\mathrm{K}^{+}$concentration in millimoles per liter. $\mathrm{B}$, The effect of arrest time on coronary vascular resistance $(C V R)$ during intermittent cardioplegic flushes (2minutes every 18 minutes) over a 2 -hour arrest period (1-hour arrest groups are not shown). $\infty, 16 \mathrm{mmol} / \mathrm{L} \mathrm{K}^{+}$alone, $P<.01$ (1-way analysis of variance) compared with $\mathrm{AL} 3.0,5.9,10$, and $16 \mathrm{mmol} / \mathrm{L} \mathrm{K}^{+}$. +, AL $3.0 \mathrm{mmol} / \mathrm{L} \mathrm{K}^{+}, P<.05$ (1-way analysis of variance) compared with AL $10 \mathrm{mmol} / \mathrm{L} \mathrm{K}^{+}$. *, AL $5.9 \mathrm{mmol} / \mathrm{L} \mathrm{K}^{+}, P<.05$ (by repeated measures) compared with $\mathrm{AL} 3$ and $16 \mathrm{mmol} / \mathrm{L} \mathrm{K}^{+}$and $16 \mathrm{mmol} /$ $\mathrm{L} \mathrm{K}^{+}$alone. $\mathrm{C}$, The effect of variation in the membrane potential on coronary vascular resistance $(C V R)$ for groups arrested for 1 and 2 hours at $32^{\circ} \mathrm{C}$ to $33^{\circ} \mathrm{C}$. The $\mathrm{AL} 0.1 \mathrm{mmol} / \mathrm{L} \mathrm{K}^{+}$group $(\varphi=-183 \mathrm{mV})$ and the $25 \mathrm{mmol} / \mathrm{L}$ $\mathrm{K}^{+}$alone group $(\varphi=-39 \mathrm{mV})$ were not included in the 2-hour arrest groups because nearly $40 \%$ failed to return aortic flow after 1hour arrest. Insert, Comparison of CVR after 1 or 2 hours' arrest with AL containing 5.9, 10 , or $16 \mathrm{mmol} / \mathrm{L} \mathrm{K} \mathrm{K}^{+}$. CVR after 1 hour's arrest was as follows: CVR = $0.0116\left[\mathrm{~K}^{+} \mathrm{mmol} / \mathrm{L}\right]+0.2004\left(R^{2}=0.78\right)$. That after 2 hours was as follows: $\mathrm{CVR}=0.0251\left[\mathrm{~K}^{+}\right]+0.1834, \mathrm{R}^{2}=0.94$. 

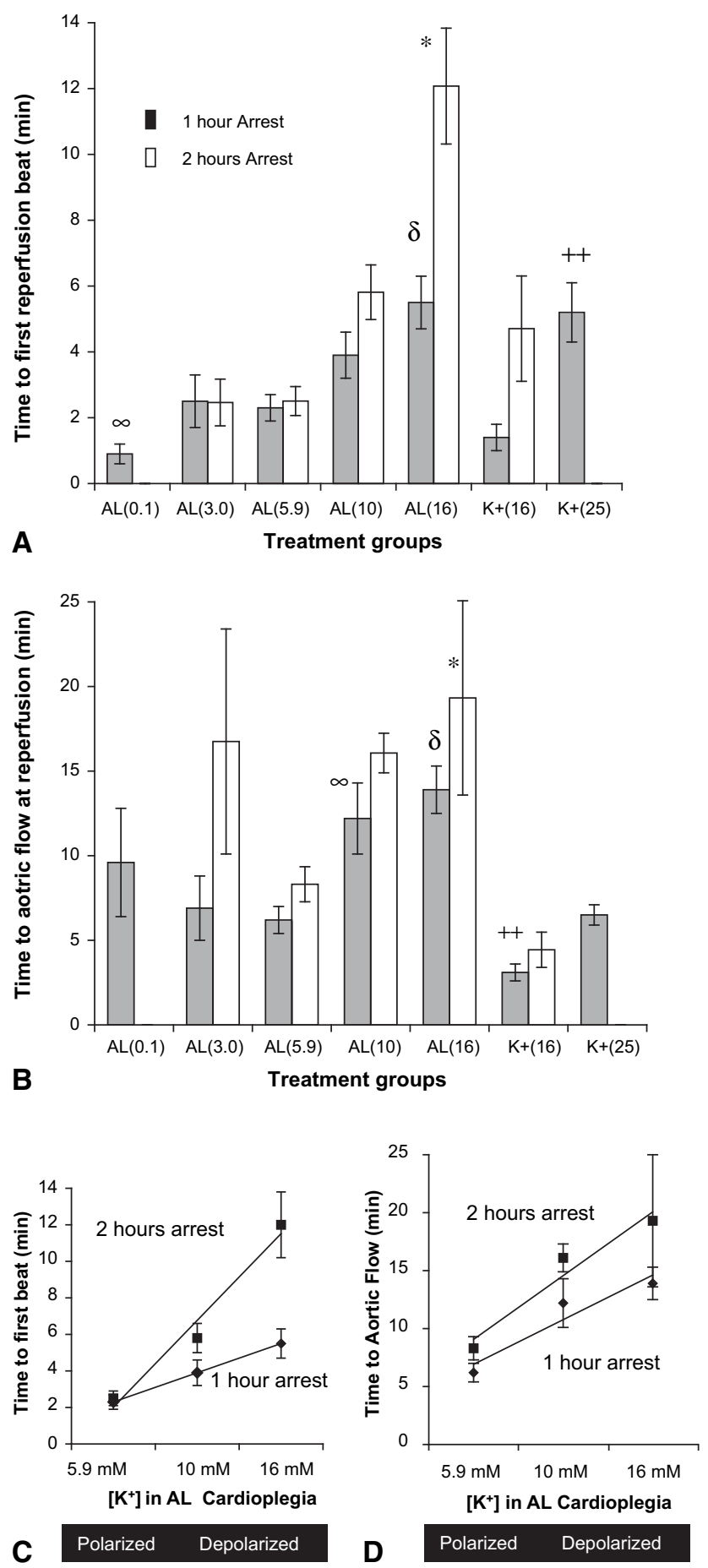

$\left[\mathrm{K}^{+}\right]$in AL Cardioplegia

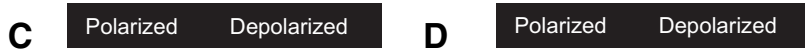

FIGURE 2. A, Time to first ventricular beat at reanimation in working mode after 1 hour's (shaded) or 2 hours' (not shaded) arrest. Adenosinelidocaine (AL) $0.1 \mathrm{mmol} / \mathrm{L}$ potassium $\left(\mathrm{K}^{+}\right)$and $25 \mathrm{mmol} / \mathrm{L} \mathrm{K}^{+}$alone were not included in the 2-hour arrest study because approximately $40 \%$ failed to recover function after 1 hour's arrest (same for panel B). $\infty$, AL 0.1 $\mathrm{mmol} / \mathrm{L} \mathrm{K}^{+}, P<.05$ compared with $\mathrm{AL} 10$ and $16 \mathrm{mmol} / \mathrm{L} \mathrm{K}{ }^{+} \delta$, AL 16 $\mathrm{mmol} / \mathrm{L} \mathrm{K}^{+}, P<.05$ compared with $\mathrm{AL} 3$ and $5.9 \mathrm{mmol} / \mathrm{L} \mathrm{K}^{+}$and 16 $\mathrm{mmol} / \mathrm{L} \mathrm{K}^{+}$alone. *, AL $16 \mathrm{mmol} / \mathrm{L} \mathrm{K}^{+}, P<.01$ compared with all other groups. +, $25 \mathrm{mmol} / \mathrm{L} \mathrm{K}^{+}$alone, $P<.05$ compared with AL 0.1 and 5.9 (percentage recovery) $=-3.8406\left[\mathrm{~K}^{+} \mathrm{mmol} / \mathrm{L}\right]+128.84\left(R^{2}\right.$ $=0.92$ ). That after 2 hours' arrest was as follows: HR (percentage recovery $)=-6.1517\left[\mathrm{~K}^{+} \mathrm{mmol} / \mathrm{L}\right]+118.08\left(R^{2}=\right.$ $0.75)$

After 1 and 2 hours' arrest, hearts in the AL $5.9 \mathrm{mmol} / \mathrm{L}$ $\mathrm{K}^{+}$group demonstrated a rapid return of systolic and diastolic pressures, with nearly $100 \%$ return at 15 minutes and $92 \%$ to $100 \%$ recoveries at 60 minutes (Table 2). After 1 hour's arrest, the $\mathrm{AL} 10 \mathrm{mmol} / \mathrm{L} \mathrm{K}^{+}$and $16 \mathrm{mmol} / \mathrm{L} \mathrm{K}^{+}$ alone groups recovered $90 \%$ to $100 \%$ developed pressures at 15 minutes; however, in contrast to the $\mathrm{AL} 5.9 \mathrm{mmol} / \mathrm{L} \mathrm{K}^{+}$ group, the $\mathrm{AL} 10 \mathrm{mmol} / \mathrm{L} \mathrm{K}^{+}$group was slow to recover after 2 hours' arrest, with only 50\% developed pressures at 15 minutes. After 2 hours, the $\mathrm{AL} 16 \mathrm{mmol} / \mathrm{L} \mathrm{K}^{+}$and 16 $\mathrm{mmol} / \mathrm{L} \mathrm{K}^{+}$alone groups recovered $43 \%$ to $66 \%$ developed pressures at 15 minutes, and the $\mathrm{AL} 3 \mathrm{mmol} / \mathrm{L} \mathrm{K}^{+}$group recovered only $17 \%$ to $26 \%$ at 15 minutes; developed pressures for these groups changed little over the next 45 minutes (Table 2).

After 1 and 2 hours' arrest, the AL $5.9 \mathrm{mmol} / \mathrm{L} \mathrm{K}^{+}$group recovered $81 \%$ to $91 \%$ of its prearrest $\mathrm{AF}$ at 60 minutes (Table 2), and nearly $100 \%$ return occurred at 15 minutes. After 2 hours' arrest, this recovery for the AL $5.9 \mathrm{mmol} / \mathrm{L}$ $\mathrm{K}^{+}$group was significantly higher than for all other groups (Table 2). After 1 hour's arrest, the AL 10, 16, and 3 $\mathrm{mmol} / \mathrm{L} \mathrm{K} \mathrm{K}^{+}$and $16 \mathrm{mmol} / \mathrm{L} \mathrm{K}^{+}$alone groups recovered $57 \%, 44 \%, 71 \%$, and $75 \%$, respectively, of prearrest AF at 15 minutes and $79 \%, 81 \%, 81 \%$, and $69 \%$ at 60 minutes (Table 2). Hearts in the $25 \mathrm{mmol} / \mathrm{L} \mathrm{K}^{+}$alone group recovered significantly less, with $43 \% \mathrm{AF}$ at 60 minutes $(49 \%$ return at 15 minutes). Hearts in the $\mathrm{AL} 0.1 \mathrm{mmol} / \mathrm{L} \mathrm{K}^{+}$group recovered $28 \%$ (15\% at 15 minutes). After 2 hours' arrest, the AL $10 \mathrm{mmol} / \mathrm{L} \mathrm{K}^{+}$group recovered $71 \%$ at 60 minutes but showed only $26 \%$ return at 15 minutes. The $16 \mathrm{mmol} / \mathrm{L} \mathrm{K}^{+}$ alone, $\mathrm{AL} 16 \mathrm{mmol} / \mathrm{L} \mathrm{K}^{+}$, and $\mathrm{AL} 3 \mathrm{mmol} / \mathrm{L} \mathrm{K}^{+}$groups recovered $33 \%, 15 \%$, and $4 \%$, respectively, of their prearrest $\mathrm{AF}$ at 15 minutes (Table 2). The relationship between AF and increasing extracellular $\mathrm{K}^{+}$concentrations in AL cardioplegia

$\mathrm{mmol} / \mathrm{L} \mathrm{K}^{+}$and $16 \mathrm{mmol} / \mathrm{L} \mathrm{K}^{+}$alone. B, Time to aortic flow at reperfusion after 1 hour's (shaded) or 2 hours' (not shaded) arrest. $\infty, \mathrm{AL} 10 \mathrm{mmol} / \mathrm{L}$ $\mathrm{K}^{+}, P<.01$ compared with $16 \mathrm{mmol} / \mathrm{L} \mathrm{K}^{+}$alone. $\delta$, $\mathrm{AL} 16 \mathrm{mmol} / \mathrm{L} \mathrm{K}{ }^{+}, P<$ .05 compared with AL 3 and $5.9 \mathrm{mmol} / \mathrm{L} \mathrm{K}^{+}$. *, AL $16 \mathrm{mmol} / \mathrm{L} \mathrm{K}{ }^{+}, P<.05$ compared with AL $5.9 \mathrm{mmol} / \mathrm{L} \mathrm{K}^{+}$and $16 \mathrm{mmol} / \mathrm{L} \mathrm{K}^{+}$alone. + , $16 \mathrm{mmol} / \mathrm{L}$ $\mathrm{K}^{+}$alone, $P<.01$ compared with AL 10 and $16 \mathrm{mmol} / \mathrm{L} \mathrm{K}^{+}$. C, Relationship between the time to first beat at reanimation after 1 or 2 hours' arrest and the concentration of $\mathrm{K}^{+}$in $\mathrm{AL}$ cardioplegic solution (5.9, 10, and $16 \mathrm{mmol} / \mathrm{L}$ $\mathrm{K}^{+}$). Time to first beat after 1hour was as follows: Time to first beat (min) $=0.3131\left[\mathrm{~K}^{\dagger}\right]+0.5703,\left(R^{2}=0.99\right)$. That after 2hours was as follows: Time to first beat $(\mathrm{min})=0.9474\left[\mathrm{~K}^{+}\right]-3.3076\left(R^{2}=0.99\right)$. D, Relationship between the time to aortic flow after 1 or 2 hours' arrest and the concentration of $\mathrm{K}^{+}$in $\mathrm{AL}$ cardioplegic solution $\left(5.9,10\right.$, and $16 \mathrm{mmol} / \mathrm{L} \mathrm{K}^{+}$). Time to aortic flow after 1hour was as follows: Time to aortic flow $(\mathrm{min})=0.727$ $\left[\mathrm{K}^{+}\right]+3.3035\left(R^{2}=0.84\right)$. That after 2hours was as follows: Time to aortic flow $(\min )=1.057\left[\mathrm{~K}^{+}\right]+3.290\left(R^{2}=0.88\right)$. 

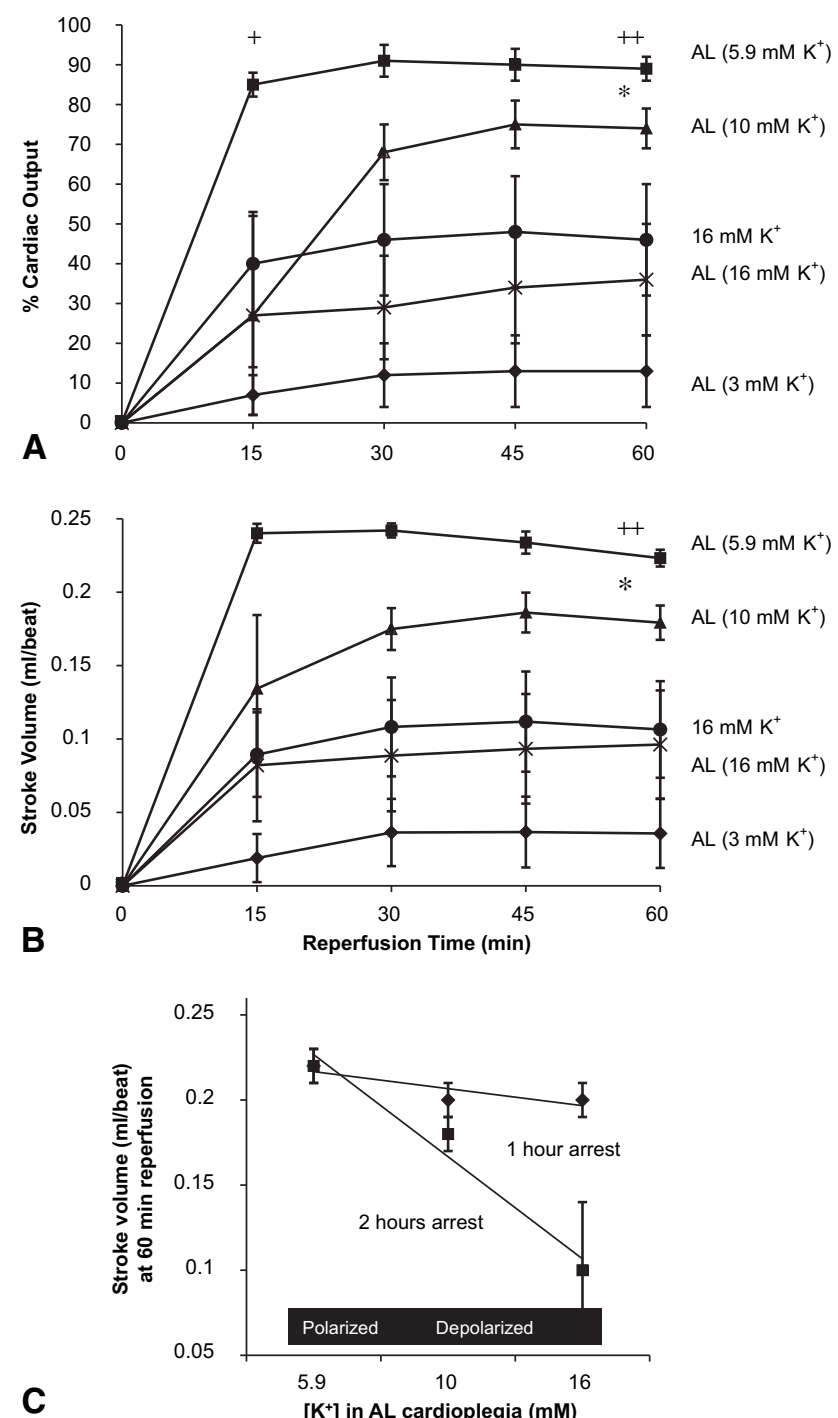

C

$\left[\mathrm{K}^{+}\right]$in AL cardioplegia ( $\mathrm{mM}$ )

FIGURE 3. A, Recovery of cardiac output (percentage of prearrest values) during 60 minutes' reperfusion after 2 hours' arrest at $32^{\circ} \mathrm{C}$ to $33^{\circ} \mathrm{C}$. + , Adenosine-lidocaine $(A L) 5.9 \mathrm{mmol} / \mathrm{L} \mathrm{K}^{+}, P<.01$ (1-way analysis of variance) compared with $\mathrm{AL} \mathrm{3,10}$, and $16 \mathrm{mmol} / \mathrm{L}$ potassium $\left(\mathrm{K}^{+}\right)$and $16 \mathrm{mmol} / \mathrm{L}$ $\mathrm{K}^{+}$alone. + , AL $5.9 \mathrm{mmol} / \mathrm{L} \mathrm{K}^{+}, P<.01$ (1-way analysis of variance) compared with $\mathrm{AL} 3 \mathrm{mmol} / \mathrm{L} \mathrm{K} \mathrm{K}^{+}, P<.05$ (repeated measures) compared with AL 3 and $16 \mathrm{mmol} / \mathrm{L} \mathrm{K}^{+}$and $16 \mathrm{mmol} / \mathrm{L} \mathrm{K}^{+}$alone. *, $\mathrm{AL} 10 \mathrm{mmol} / \mathrm{L} \mathrm{K}^{+}, P$ $<.01$ (repeated measures) compared with $\mathrm{AL} 3 \mathrm{mmol} / \mathrm{L} \mathrm{K}^{+}$. B, Recovery of stroke volume (in milliliters per beat) during 60 minutes' reperfusion after 2 hours' arrest at $32^{\circ} \mathrm{C}$ to $33^{\circ} \mathrm{C}$. + , $\mathrm{AL} 5.9 \mathrm{mmol} / \mathrm{L} \mathrm{K}^{+}, P<.01$ (repeated measures) compared with $\mathrm{AL} 3$ and $16 \mathrm{mmol} / \mathrm{L} \mathrm{K}^{+}$and $16 \mathrm{mmol} / \mathrm{L} \mathrm{K}^{+}$alone. *, $\mathrm{AL}$ $10 \mathrm{mmol} / \mathrm{L} \mathrm{K}^{+}, P<.01$ (repeated measures) compared with $\mathrm{AL} 3 \mathrm{mmol} / \mathrm{L} \mathrm{K}^{+}$. $\mathrm{C}$, Relationship between stroke volume and increasing concentrations of $\mathrm{K}^{+}$in AL cardioplegic solution $\left(5.9,10\right.$, and $\left.16 \mathrm{mmol} / \mathrm{L} \mathrm{K}^{+}\right)$at 60 minutes' reperfusion for 1- and 2-hour arrest groups. Stroke volume after 1hour was as follows: Stroke volume $=0.0018\left[\mathrm{~K}^{+} \mathrm{mmol} / \mathrm{L}\right]+0.2262\left(R^{2}=0.65\right)$. That after 2 hours was as follows: Stroke volume $=0.012\left[\mathrm{~K}^{\dagger}\right]+0.294\left(R^{2}=0.99\right)$.

at 15 minutes' reperfusion after 1 hour's arrest was as follows: $\mathrm{AF}$ (percentage recovery) $=-4.4704\left[\mathrm{~K}^{+} \mathrm{mmol} / \mathrm{L}\right]+$ $111.53\left(R^{2}=0.88\right)$. That after 2 hours' arrest was as follows:
$\mathrm{AF}($ percentage recovery $)=-6.1885\left[\mathrm{~K}^{+} \mathrm{mmol} / \mathrm{L}\right]+106.47$ $\left(R^{2}=0.79\right)$.

Recovery of rate and percentage of $\mathrm{CF}$ showed profiles similar to those of AF (Table 2). After 1 and 2 hours' arrest, the $\mathrm{AL} 5.9 \mathrm{mmol} / \mathrm{L} \mathrm{K}^{+}$group recovered $101 \%$ to $113 \%$ of its prearrest $\mathrm{CF}$ at 60 minutes, and $95 \%$ of this recovery was achieved at 15 minutes' reperfusion. After 1 hour's arrest, the $\mathrm{AL} 10,16$, and $3 \mathrm{mmol} / \mathrm{L} \mathrm{K}^{+}$and $16 \mathrm{mmol} / \mathrm{L} \mathrm{K}^{+}$ alone groups recovered $72 \%, 65 \%, 72 \%$, and $78 \%$, respectively, of prearrest $\mathrm{CF}$ at 15 minutes and $97 \%, 94 \%, 90 \%$, and $81 \%$ at 60 minutes' reperfusion. Hearts arrested with 25 $\mathrm{mmol} / \mathrm{L} \mathrm{K}^{+}$alone recovered only $54 \%$ (49\% at 15 minutes), and those arrested with $\mathrm{AL} 0.1 \mathrm{mmol} / \mathrm{L} \mathrm{K}^{+}$recovered $41 \%$ ( $45 \%$ at 15 minutes) of their prearrest CF. After 2 hours' arrest, the $\mathrm{AL} 10 \mathrm{mmol} / \mathrm{L} \mathrm{K}^{+}$group recovered $81 \%$ at $60 \mathrm{~min}-$ utes, and only $40 \%$ of recovery occurred at 15 minutes. The $16 \mathrm{mmol} / \mathrm{L} \mathrm{K}^{+}$alone, AL $16 \mathrm{mmol} / \mathrm{L} \mathrm{K}^{+}$, and $\mathrm{AL} 3.0 \mathrm{mmol} / \mathrm{L}$ $\mathrm{K}^{+}$groups recovered $44 \%, 29 \%$, and $15 \%$ of their prearrest CF at 15 minutes' and $49 \%, 33 \%$, and $17 \%$ at 60 minutes' reperfusion, respectively (Table 2). The relationship between $\mathrm{CF}$ and increasing extracellular $\mathrm{K}^{+}$concentrations in AL cardioplegia at 15 minutes' reperfusion after 1 hour's arrest was as follows: $\mathrm{CF}$ (percentage recovery) $=-2.8375$ $\left[\mathrm{K}^{+} \mathrm{mmol} / \mathrm{L}\right]+107.51\left(R^{2}=0.84\right)$. That after 2 hours' arrest was as follows: $\mathrm{CF}$ (percentage recovery) $=-6.1885\left[\mathrm{~K}^{+}\right.$ $\mathrm{mmol} / \mathrm{L}]+120.47\left(R^{2}=0.79\right)$.

The recovery of $\mathrm{CO}$ and $\mathrm{SV}$ are shown in Table 2 and Figure 3. After 1 and 2 hours' arrest, the AL $5.9 \mathrm{mmol} / \mathrm{L} \mathrm{K}^{+}$ group recovered $89 \%$ to $93 \%$ of its prearrest $\mathrm{CO}$ at $60 \mathrm{~min}-$ utes $(85 \%$ to $93 \%$ of this recovery at 15 minutes; Figure 3 , A). After 1 hour's arrest, the $\mathrm{AL} 10,16,3 \mathrm{mmol} / \mathrm{L} \mathrm{K}^{+}$and 16 $\mathrm{mmol} / \mathrm{L} \mathrm{K}^{+}$alone groups recovered $61 \%, 49 \%, 71 \%$, and $76 \%$ of prearrest $\mathrm{CO}$ at 15 minutes' and $84 \%, 84 \%, 83 \%$ and $72 \%$ at 60 minutes' reperfusion, respectively. Hearts arrested with $25 \mathrm{mmol} / \mathrm{L} \mathrm{K}^{+}$alone recovered only $46 \%(49 \%$ at 15 minutes), and those arrested with $\mathrm{AL} 0.1 \mathrm{mmol} / \mathrm{L} \mathrm{K}^{+}$recovered $32 \%$ prearrest $\mathrm{CO}$. The relationship between $\mathrm{CO}$ and increasing extracellular $\mathrm{K}^{+}$concentrations in AL cardioplegia at 15 minutes' reperfusion after 1 hour's arrest was as follows: $\mathrm{CO}$ (percentage recovery) $=-4.1829\left[\mathrm{~K}^{+} \mathrm{mmol} / \mathrm{L}\right]$ $+112.15\left(R^{2}=0.87\right)$. After 2 hours' arrest, the AL $10 \mathrm{mmol} /$ $\mathrm{L} \mathrm{K}^{+}$group recovered $74 \%$ of prearrest value and $27 \%$ at 15 minutes' reperfusion, which was significantly less than that seen in the AL $5.9 \mathrm{mmol} / \mathrm{L} \mathrm{K}^{+}$group. The $16 \mathrm{mmol} / \mathrm{L} \mathrm{K}^{+}$ alone, $\mathrm{AL} 16 \mathrm{mmol} / \mathrm{L} \mathrm{K}^{+}$, and $\mathrm{AL} 3.0 \mathrm{mmol} / \mathrm{L} \mathrm{K}^{+}$groups recovered $40 \%, 27 \%$, and $7 \%$ of prearrest CO after 15 minutes' and $46 \%, 36 \%$, and $13 \%$ at 60 minutes' reperfusion, respectively (Figure $3, A$, and Table 2 ). Similar recovery profiles were found with SV (Figure 3,B). After 2 hours' arrest, the AL $5.9 \mathrm{mmol} / \mathrm{L} \mathrm{K}^{+}$group recovered $0.22 \pm 0.1 \mathrm{~mL} /$ beat ( $84 \%$ of prearrest SV) and $0.24 \pm 0.1 \mathrm{~mL} /$ beat $(90 \%$ recovery was achieved at 15 minutes). This recovery was significantly higher than that seen in the AL $3 \mathrm{mmol} / \mathrm{L} \mathrm{K}^{+}$, AL 16 $\mathrm{mmol} / \mathrm{L} \mathrm{K}^{+}$, and $16 \mathrm{mmol} / \mathrm{L} \mathrm{K}^{+}$alone groups. 


\section{DISCUSSION}

Surgeons have known for decades that depolarizing cardioplegia does not afford optimal cardioprotection. ${ }^{2,3}$ A number of years ago, we introduced an alternative arrest, protection, and preservation concept using nondepolarizing normokalemic AL. ${ }^{7,8,12}$ The aim of the present study was to examine the effects of varying the concentration of extracellular $\mathrm{K}^{+}$in $\mathrm{AL}$ cardioplegia on arrest and reanimation properties in the isolated working rat heart at warm temperatures. We show that warm AL cardioplegia is most efficient under normokalemic conditions when the myocardial cell membrane potential is close to its resting state. Hearts arrested with lower $\mathrm{K}^{+}$concentrations (hyperpolarizing cardioplegia) or higher $\mathrm{K}^{+}$concentrations (depolarizing cardioplegia) had significantly higher CVRs during arrest, experienced reanimation arrhythmias, and were slow to recover, with significant losses in CO, SV, and contractility. Nearly $40 \%$ of hearts in the $\mathrm{AL} 0.1 \mathrm{mmol} / \mathrm{L} \mathrm{K}^{+}$and $25 \mathrm{mmol} / \mathrm{L} \mathrm{K}^{+}$alone groups failed to return $\mathrm{HR}$, developed pressures, or $\mathrm{CO}$ after 1 hour's arrest.

\section{Effect of Changing Extracellular $\mathrm{K}^{+}$Concentrations on Cardiac Membrane Potential}

The theoretic relationship between resting membrane potential and transmembrane $\mathrm{K}^{+}$concentration gradient was first proposed in 1902 by German physiologist Julius Bernstein, ${ }^{18}$ after Ostwald and Nernst. We report a Nernstian relationship between changing $\mathrm{K}^{+}$concentrations and membrane potential $(\varphi)$ in the arrested heart: $\varphi(\mathrm{mV})=$ $26.23 \ln \left[\mathrm{K}^{+} \mathrm{mmol} / \mathrm{L}\right]-123.44\left(\mathrm{R}^{2}=0.99\right.$; Figure $\left.1, A\right)$. This finding is in good agreement with our previous work on the rat heart ${ }^{12,19}$ and with the large number of independent microelectrode measurements made on in vivo, isolated heart and myocyte preparations. ${ }^{4,20,21}$ For example, Snabaitis and colleagues ${ }^{4}$ measured $-50 \mathrm{mV}$ in the isolated rat heart perfused with $16 \mathrm{mmol} / \mathrm{L} \mathrm{K}^{+}$alone in $\mathrm{K}-\mathrm{H}$ buffer $(\mathrm{n}=8)$, and Kleber ${ }^{20}$ measured $-82 \pm 2 \mathrm{mV}$ in the isolated guinea pig heart perfused with $4.5 \mathrm{mmol} / \mathrm{L} \mathrm{K}^{+}(\mathrm{n}=6)$. From Figure $1, A$, our values for Snabaitis and colleagues ${ }^{4}$ and Kleber's ${ }^{20}$ measurements are -50.7 and $-84 \mathrm{mV}$, respectively, at identical $\mathrm{K}^{+}$concentrations. Similarly, at lower $\mathrm{K}^{+}$concentrations $(3.0 \mathrm{mmol} / \mathrm{L})$, Wan and coworkers ${ }^{21}$ measured $-93 \mathrm{mV}$ using microelectrodes in ventricular myocytes of guinea pigs, and our value from Figure $1, A$, is $-94 \mathrm{mV}$ and in good agreement given the limitations of both methods. In the present study we did not estimate the membrane potential throughout the arrest period but only at induction. Snabaitis and colleagues ${ }^{4}$ showed that membrane potential remained relatively constant during polarized and nonpolarized arrest time in the rat heart receiving tetrodotoxin and $16 \mathrm{mmol} / \mathrm{L} \mathrm{K}$ solution, respectively. It would be of interest to conduct future studies with both microelectrode measurements and Nernstian $\mathrm{K}^{+}$distributions during arrest induction, maintenance, and recovery. Because the Nernst equation strictly describes an equilibrium state, our data lend support to the concept that the resting membrane potential in the heart is not a diffusion potential but arises from a measure of electrical work. ${ }^{6,22}$ Our data indicate that the net myocardial transmembrane flux of $\mathrm{K}^{+}$over the range of 0.1 to $25 \mathrm{mmol} / \mathrm{L} \mathrm{K}^{+}$is zero, which means during diastolic arrest, the net inward current (eg, sum of rectifier IK1) ${ }^{23}$ is equal to the net reversal potential for $\mathrm{K}^{+}$ions where $\triangle \mathrm{G}^{\prime}$ $\left[\mathrm{K}^{\dagger}\right]_{\mathrm{o} / \mathrm{i}}=0 .{ }^{19,22}$ In conclusion, despite decreasing the transmembrane gradient by increasing extracellular $\mathrm{K}^{+}$concentrations from 5.9 to $25 \mathrm{mmol} / \mathrm{L}$ (increasing depolarization), as is widely used to induce arrest in cardiac surgery, our study indicates that the heart membrane potential follows a Nernstian relationship.

\section{Effect of Changing $\mathrm{K}^{+}$and Membrane Potential on CVR During Arrest}

Hypokalemia. We showed that hypokalemic AL arrest $\left(0.1\right.$ and $\left.3.0 \mathrm{mmol} / \mathrm{L} \mathrm{K}^{+}\right)$led to significantly higher CVR values (up to 2.6-fold after 2 hours' arrest) compared with AL normokalemia (Figure 1, B). In 1974, Brace and associates $^{24}$ reported that an intracoronary hypokalemic (2.1 $\mathrm{mmol} / \mathrm{L} \mathrm{K}^{+}$) infusion into the canine heart in vivo under constant pressure led to reduced blood flow and increased oxygen consumption. They concluded that the increase in CVR was either the result of active vasoconstriction (direct vascular smooth muscle contraction), passive vasoconstriction (extravascular compression), or both.

Normokalemia. The CVR for AL cardioplegia with 5.9 $\mathrm{mmol} / \mathrm{L} \mathrm{K}^{+}$increased by only $30 \%$ over 2 hours' warm arrest $\left(0.24\right.$ to $0.31 \pm 0.01$ megadyne $\left.\cdot \mathrm{s}^{-1} \cdot \mathrm{cm}^{-5}, \mathrm{n}=16\right)$. Brace and associates ${ }^{24}$ reported that a small $\mathrm{K}^{+}$window exists between approximately 5 and $10 \mathrm{mmol} / \mathrm{L}$ at which reduced CVR and dilation occurs; however, we did not investigate this window in the present study.

Hyperkalemia. For many decades, hyperkalemia has been linked to increased CVR, endothelial dysfunction, and vasospasm. ${ }^{9,25,26} \mathrm{We}$ confirmed our earlier results in the isolated rat heart showing that hyperkalemia increases CVR during warm arrest ${ }^{8,12}$ and that CVR was linearly related to extracellular $\mathrm{K}^{+}$in $\mathrm{AL}$ cardioplegia (from 5.9 to 16 $\mathrm{mmol} / \mathrm{L}$; Figure $1, B$, insert). It is noteworthy that the rate of change in CVR with increasing $\mathrm{K}^{+}$(slope) was more than 2 times higher with doubling of arrest time (Table 1 and Figure 1, $B$, insert). In terms of membrane potential, hyperpolarization $(-183$ and $-94 \mathrm{mV})$ or depolarization $(-65,-49$, and $-39 \mathrm{mV})$ increases CVR to a significantly greater extent than does normokalemic AL membrane polarization (Figure 1,C). We conclude that diastolic arrest voltages outside the normal physiologic limits have an untoward effect on CVR and might reduce cardioplegia distribution and compromise protection during surgical arrest and reanimation. 


\section{Time to First Beat and AF and Reperfusion Arrhythmias}

Time to first spontaneous ventricular beat also increased linearly with extracellular $\mathrm{K}^{+}$concentrations in $\mathrm{AL}$ cardioplegia (Figure 2, $A$ and $C$ ). After 1 hour, time to first beat increased from 2.3 to 5.5 minutes, and after 2 hours, it increased from 2.5 to 12 minutes (Figure 2, $A$ and $C$ ). Interestingly, the difference in slopes in Figure 2, $C$, shows that time to first spontaneous ventricular beat (in minutes) per millimole per liter of $\mathrm{K}^{+}$is 3 times longer after 2 hours' arrest and importantly that time to first spontaneous ventricular beat for AL $\left(5.9 \mathrm{mmol} / \mathrm{L} \mathrm{K}^{+}\right)$was unchanged (Figure 2,C). Similar relations were found for time to first $\mathrm{AF}$ (Figure 2, $B$ and $D$ ). Increasing $\mathrm{K}^{+}$concentrations also increased the incidence of abnormal heart rhythms during reanimation. Hearts arrested with $\mathrm{AL} 5.9 \mathrm{mmol} / \mathrm{L} \mathrm{K}^{+}$ cardioplegia for 1 and 2 hours' arrest were the only groups in which all hearts showed continual and consistent spontaneous beating with no arrhythmias in early reperfusion (Data not shown).

\section{Recovery of Pump Function}

Hearts arrested with nornokalemic AL $\left(5.9 \mathrm{mmol} / \mathrm{L} \mathrm{K}^{+}\right)$ cardioplegia were also superior in functional recovery of $\mathrm{HR}$, developed pressures, $\mathrm{AF}, \mathrm{CF}, \mathrm{CO}$, and $\mathrm{SV}$ compared with those hearts receiving hypokalemic or hyperkalemic AL cardioplegia or 16 and $25 \mathrm{mmol} / \mathrm{L} \mathrm{K}^{+}$alone cardioplegia (Table 2 and Figure 3).

We found that after 1 and 2 hours' warm arrest, the AL 5.9 $\mathrm{mmol} / \mathrm{L} \mathrm{K}^{+}$cardioplegia groups recovered rapidly and predictably $90 \%$ to $110 \%$ of $\mathrm{HR}$, developed pressures, $\mathrm{AF}$, $\mathrm{CF}$, and $\mathrm{CO}$ (Table 2 and Figure 3, A). After 2 hours, hearts receiving depolarizing $\mathrm{AL} 10 \mathrm{mmol} / \mathrm{L} \mathrm{K}^{+}$cardioplegia recovered function slower, with significantly lower $\mathrm{CO}$ at 15 minutes' reperfusion (Table 2 and Figure $3, A$ ). Increasing the $\mathrm{K}^{+}$concentration to $16 \mathrm{mmol} / \mathrm{L}$ in $\mathrm{AL}$ cardioplegia led to further functional loss in recovery of $\mathrm{HR}$, developed pressures, $\mathrm{AF}, \mathrm{CF}$, and $\mathrm{CO}$ (Table 2 and Figure 3, A). Interestingly, nearly all metrics changed in a linear fashion with increasing $\mathrm{K}^{+}$concentrations (from 5.9 to $16 \mathrm{mmol} / \mathrm{L}$ ) at 15 and 60 minutes' reperfusion (Table 2 and Figure 3). Furthermore, doubling the arrest time exacerbated the damaging effects of $\mathrm{K}^{+}$(see the Results section). The lower functional recoveries for the $\mathrm{AL} 10$ and $16 \mathrm{mmol} / \mathrm{L} \mathrm{K}^{+}$or 16 and 25 $\mathrm{mmol} / \mathrm{L} \mathrm{K}^{+}$alone groups might be due to $\mathrm{K}^{+}$-induced myocardial (and vascular) stunning through $\mathrm{H}^{+}$ion imbalances, oxidative stress, calcium overload, and mitochondrial oxidative dysfunction. ${ }^{11}$

The effect of increasing $\mathrm{K}^{+}$concentrations in AL cardioplegia on loss of functional recovery was highlighted by a decrease of up to $67 \%$ in SV (Figure 3, B). SV (in milliliters per beat) was calculated by dividing $\mathrm{CO}$ by $\mathrm{HR}$, and because the preload $\left(10 \mathrm{~cm} \mathrm{H}_{2} \mathrm{O}\right)$ and afterload $\left(100 \mathrm{~cm} \mathrm{H}_{2} \mathrm{O}\right)$ are preset in the working heart preparation, a decrease in $\mathrm{SV}$ might indicate a decrease in left ventricular contractility. The relationship between $\mathrm{SV}$ and increasing $\mathrm{K}^{+}$concentrations in the AL cardioplegia after 1 hour's arrest at 15 minutes' reperfusion was as follows: $\mathrm{SV}(\mathrm{mL} /$ beat $)=-0.0085$ $\left[\mathrm{K}^{+} \mathrm{mmol} / \mathrm{L}\right]+0.2771\left(R^{2}=0.84\right)$. That after 2 hours was as follows: $\mathrm{SV}(\mathrm{mL} / \mathrm{beat})=-0.0152\left[\mathrm{~K}^{+} \mathrm{mmol} / \mathrm{L}\right]+$ $0.3103\left(R^{2}=0.88\right)$. After 2 hours, SV decreased by up to $67 \%$ at 15 minutes' and $60 \%$ at 60 minutes' reperfusion, and the rate of decrease was more than 10-fold compared with that seen in the 1-hour arrest group at 60 minutes' reperfusion (Figure 3, $B$ and $C$ ). We conclude that increasing $\mathrm{K}^{+}$ concentrations from 5.9 to 10 and $16 \mathrm{mmol} / \mathrm{L}$ in AL cardioplegia results in a significant loss of $\mathrm{CO}, \mathrm{SV}$, and left ventricular contractility, and the decrease is more pronounced the longer the arrest time. Pressure-volume loops or an intraventricular balloon would be required to quantify the effects of $\mathrm{AL}$ with varying $\mathrm{K}^{+}$concentrations on changes in contractility (eg, degree of sarcomere stretch) during recovery. In cell voltage terms the more the cardiomyocyte, heart conduction cells, and cells of the coronary vasculature appear to deviate from their natural resting membrane potentials during cardioplegic arrest, the greater the loss of cardiac function during arrest, reanimation, and reperfusion.

\section{Possible Clinical Significance: Polarized Versus Depolarized Cardioplegia}

Although the underlying mechanisms of AL likely involve an interaction of adenosine receptors, $\mathrm{Na}^{+}$fast channels, protein kinase $\mathrm{C}$ survival pathways, and adenosine triphosphate-sensitive $\mathrm{K}^{+}$channels (mitochondrial and sarcolemmal), ${ }^{12}$ the key to polarized arrest and related polarizing cardioprotection strategies is that there are fewer voltage-dependent membrane channels, pores, and exchangers $\left(\mathrm{Na}^{+} / \mathrm{H}^{+}\right.$and $\left.\mathrm{Na}^{+} / \mathrm{Ca}^{2+}\right)$ open compared with depolarized states. ${ }^{5,12}$ Depolarizing $\mathrm{K}^{+}$cardioplegia has been a doubleedged sword in cardiac surgery for more than 4 decades, particularly after Tyers and colleagues ${ }^{27}$ demonstrated unequivocally that $\mathrm{K}^{+}$, and not citrate, was responsible for the clinical failure of Melrose's method. Currently, there are at least 5 clinical concerns with depolarizing $\mathrm{K}^{+}$cardioplegia. These include (1) generating unnatural voltages and ionic gradients in the heart leading to cell $\mathrm{Na}^{+}$and $\mathrm{Ca}^{2+}$ loading, ${ }^{11}$ which are exacerbated with longer crossclamp times $^{7,12}$; (2) coronary vasoconstriction leading to higher resistances, suboptimal delivery pressure-flow relations, and nonuniform distribution of cardioplegia ${ }^{28,29}$; (3) damage to the vascular endothelium and smooth muscle interactions, which predispose the coronary vessels to $\operatorname{spasm}^{9,30}$; (4) the occurrence of postoperative atrial and ventricular arrhythmias, including conduction disturbances ${ }^{10}$; and (5) ventricular dysfunction, such as postcardioplegia stunning. ${ }^{11}$

Because polarizing normokalemic AL cardioplegia maintains cell voltages close to resting values, this strategy might offer surgeons an alternative and address the growing 
concern about the lack of cardioprotection in high-risk patients undergoing cardiac surgery or those with extended crossclamp times. Although the present study was conducted on healthy hearts, future studies need to be conducted on older hearts with comorbidities and neonatal hearts. A few clinical data are available on crystalloid and all-blood AL cardioplegia in pediatric and adult cardiac surgery. Jin and associates ${ }^{31}$ reported that a "one shot" of AL 10 $\mathrm{mmol} / \mathrm{L} \mathrm{K} \mathrm{K}^{+}$crystalloid cardioplegia was more protective than $\mathrm{AL} 20 \mathrm{mmol} / \mathrm{L} \mathrm{K}^{+}$or non-AL $20 \mathrm{mmol} / \mathrm{L} \mathrm{K}^{+}$cardioplegia alone in 134 pediatric patients undergoing correction of ventricular septal defect. With decreasing $\mathrm{K}^{+}$concentrations, these authors showed that as the membrane potential shifted toward more polarized levels (ie, from -46 to $-67 \mathrm{mV}$ ), there was (1) a reduced use of inotropes, (2) a significantly lower release of troponin I after surgical intervention, and (3) 1 day less of hospitalization. ${ }^{31}$ Our data in the present study help to explain some of the advantages of physiologic levels of $\mathrm{K}^{+}$in AL cardioplegia.

Another clinical case study using all-blood AL (or Adenocaine; Hibernation Therapeutics, Wulguru, Australia) cardioplegia was reported for a $4 \times$ redo high-risk patient with infective endocarditis requiring mitral and aortic value replacements with aortic root enlargement. During cardiopulmonary bypass $(9.8$ hours, with a total arrest time of 7 hours), the patient's heart was kept quiescent with $72 \mathrm{~L}$ of all-blood Adenocaine microplegia (with $2 \mathrm{mmol} / \mathrm{L} \mathrm{K}^{+}$) for nearly 5 hours. The patient required no balloon pump and a low level of inotropic support, was extubated in 12 hours, and was transferred from the intensive care unit after 48 hours with a subsequent uneventful recovery. ${ }^{32}$ Another possible advantage for normokalemic AL cardioplegia would be in patients with renal disease or those receiving dialysis who cannot tolerate fluctuations in plasma $\mathrm{K}^{+}$concentrations.

We thank Donna Rudd and Professor Jakob Vinten-Johansen for comments on the manuscript.

\section{References}

1. Maganti M, Rao V, Armstrong S, Feindel CM, Scully HE, David TE. Redo valvular surgery in elderly patients. Ann Thorac Surg. 2009;87:521-5.

2. Cohen NM, Damiano RJ, Wechsler AS. Is there an alternative to potassium arrest? Ann Thorac Surg. 1995;60:858-63.

3. Allen BS. Pediatric myocardial protection: where do we stand? J Thorac Cardiovasc Surg. 2004;128:11-3.

4. Snabaitis AK, Shattock MJ, Chambers DJ. Comparison of polarized and depolarized arrest in the isolated rat heart for long-term preservation. Circulation. 1997; 96:3148-56.

5. Chambers DJ, Hearse DJ. Cardioplegia and surgical ischaemia. In: Sperelakis N, Kurachi Y, Terzic A, Cohen MV, eds. Heart physiology and pathophysiology. San Diego: Academic Press; 2001.p.887-926.

6. Dobson GP. Organ arrest, protection and preservation: natural hibernation to cardiac surgery: a review. Comp Biochem Physiol Part B. 2004;139:469-85.

7. Sloots K, Vinten-Johansen J, Dobson GP. Warm non-depolarising adenosinelidocaine cardioplegia: continuous versus intermittent delivery. J Thorac Cardiovasc Surg. 2007;133:1171-8.
8. Rudd DM, Dobson GP. Towards a new cold and warm non-depolarising, normokalemic arrest paradigm for orthotopic heart transplantation. J Thorac Cardiovasc Surg. 2009;137:198-207.

9. Ruel M, Khan TA, Voisine P, Bianchi C, Sellke FW. Vasomotor dysfunction after cardiac surgery. Eur J Cardiothorac Surg. 2004;26:1002-14.

10. Ellis RJ, Mavroudis C, Gardner C, Turley K, Ullyot D, Ebert PA. Relationship between atrioventricular arrhythmias and the concentration of $\mathrm{K}^{+}$ion in cardioplegic solution. J Thorac Cardiovasc Surg. 1980;80:517-26.

11. Spinale FG. Cellular and molecular therapeutics targets for treatment of contractile dysfunction after cardioplegic arrest. Ann Thorac Surg. 1999;68:1934-41.

12. Dobson GP, Jones MW. Adenosine and lignocaine: a new concept in non-depolarising surgical arrest, protection and preservation. $J$ Thorac Cardiovasc Surg. 2004; 127:794-805

13. Corvera JS, Kin H, Dobson GP, Kerendi F, Halkos ME, Katzmark S, et al. Polarized arrest with warm or cold adenosine/lidocaine blood cardioplegia is equivalent to hypothermic potassium blood cardioplegia. J Thorac Cardiovasc Surg. 2005; 129:599-606.

14. Canyon SJ, Dobson GP. Protection against ventricular arrhythmias and cardiac death using adenosine and lidocaine during regional ischemia in the in vivo rat Am J Physiol Heart Circ Physiol. 2004;287:H1286-95.

15. Homeister JW, Hoff PT, Fletcher DD, Lucchesi BR. Combined adenosine and lidocaine administration limits myocardial reperfusion injury. Circulation. 1990;82:595-608.

16. Vander Heide RS, Reimer KA. Effect of adenosine therapy at reperfusion on myocardial infarct size in dogs. Cardiovasc Res. 1996;31:711-8.

17. Mahaffey KW, Puma JA, Barbagelata NA, DiCarli MF, Leesar MA, Browne KF et al. Adenosine as an adjunct to Thrombolytic Therapy for acute myocardial infarction. J Am Coll Cardiol. 1999;34:1711-20.

18. Bernstein J. Untersuchungen zur thermodynamie der biolectrischen strome, erster theil. Pflugers Arch. 1902;92:521-62.

19. Masuda T, Dobson GP, Veech RL. The Gibbs-Donnan near-equilibrium system of heart. J Biol Chem. 1990;265:20321-34.

20. Kleber AG. Resting membrane potential, extracellular potassium activity, and in tracellular sodium activity during acute global ischemia in isolated perfused guinea pig hearts. Circ Res. 1983;52:442-50.

21. Wan X, Bryant SM, Hart G. The effects of $[\mathrm{K}+]$ o on regional differences in electrical characteristics of ventricular myocytes in guinea-pig. Exp Physiol. 2000;85 769-74.

22. Veech RL, Kashiwaya Y, King MT. The resting membrane potential of cells are measures of electrical work, not of ionic currents. Integr Physiol Behav Sci. 1995; 30:283-307.

23. Lopatin AN, Nichols CG. Inward rectifiers in the heart: an update on IK1. $J$ Mol Cell Cardiol. 2001;33:625-38.

24. Brace RA, Anderson DK, Chen WT, Scott JB, Haddy FJ. Local effects of hypokalemia on coronary resistance and myocardial contractile force. Am J Physiol. 1974;227:590-7

25. Mankad PS, Chester AH, Yacoub MH. Role of potassium concentration in cardioplegic solutions in mediating endothelial damage. Ann Thorac Surg. 1991;51: 89-93.

26. Ericsson AB, Takeshima S, Vaage J. Warm or cold continuous blood cardioplegia provides similar myocardial protection. Ann Thorac Surg. 1999;68:454-9.

27. Tyers GF, Todd GJ, Niebauer IM, Manley NJ, Waldhausen JA. The mechanism of myocardial damage following potassium citrate (Melrose) cardioplegia. Surgery. 1975;78:45-53.

28. Leicher FG, Magrassi P, LaRaia PJ, Derkac WM, Buckley MJ, Austen WG. Blood cardioplegia delivery. Deleterious effects of potassium versus lidocaine. Ann Surg. 1983;198:266-72.

29. Ozeki T, Kwon MH, Gu J, Collins MJ, Brassil J, Miller MBJ, et al. Heart preservation using continuous ex vivo perfusion improves viability and functional recovery. Circ J. 2007;71:153-9.

30. Ferguson ER, Spruell RD, Vicente WVA, Murrah CP, Holman WL. Coronary vascular regulation during post-cardioplegia reperfusion. $J$ Thorac Cardiovasc Surg. 1996;112:1054-63.

31. Jin ZX, Zhang SL, Wang XM, Bi SH, Xin M, Zhou JJ, et al. The myocardial protective effects of a moderate potassium adenosine-lidocaine cardioplegia in pediatric cardiac surgery. $J$ Thorac Cardiovasc Surg. 2008;136:1450-5.

32. O'Rullian JJ, Clayson SE, Peragallo R. Excellent outcomes in a critical redo valve replacement patient requiring ten hours of cardiopulmonary bypass and seven hours of cardioplegia using microplegia with adjunctive adenosine and lidocaine (Adenocaine ${ }^{\mathrm{TM}}$ ). J Extra Corpor Technol. 2008;40:203-5. 


\section{Appendix 1}

\section{Estimation of Myocardial Membrane Potential}

Left ventricular tissue of prearrest and arrested hearts at induction was freeze-clamped at liquid $\mathrm{N}_{2}$ temperatures, ground in a mortar, and stored at $-80^{\circ} \mathrm{C}$ until used. Samples of tissue (50-100 $\mathrm{mg}$ ) were digested with nitric acid and analyzed for total tissue $\mathrm{K}^{+}$concentrations $(\mathrm{mg} / \mathrm{kg})$ with a Varian Liberty Series II Inductively Coupled Plasma Atomic Emission Spectrometer (Melbourne, Australia).

Membrane potential $\left(\mathrm{V}_{\mathrm{M}}\right.$ or $\varphi$ in $\left.\mathrm{mV}\right)$ was calculated from the Nernstian distribution of $\mathrm{K}^{+}$ion between the extracellular and intracellular phases (equation 1).

$$
V_{M}=E_{K^{+}}=\frac{R T}{Z_{K^{+}} F} \times \ln \frac{\left[K^{+}\right]_{\mathrm{OUT}}}{\left[K^{+}\right]_{\mathrm{IN}}}
$$

where $R$ is the universal gas constant $\left(8.31 \mathrm{~J} \mathrm{~mol}^{-1} \mathrm{~K}^{-1}\right), F$ is Faraday's constant $\left(96.49 \mathrm{KJ} \mathrm{mol}^{-1} \mathrm{~V}^{-1}\right), T$ is absolute temperature $(305.15 \mathrm{~K}), z$ is the valence of $\mathrm{K}^{+}$ion $(+1)$, and $\left[\mathrm{K}^{\dagger}\right]_{\mathrm{IN}}$ and $\left[\mathrm{K}^{\dagger}\right]_{\text {OUT }}$ are the intracellular and extracellular concentrations of the $\mathrm{K}^{+}$ion in moles per liter, respectively. ${ }^{12}$ The $\left[\mathrm{K}^{\dagger}\right]_{\mathrm{IN}}$ was calculated from the following equation: $\left[\mathrm{K}^{+}\right]_{\mathrm{TOTAL}}=\mathrm{x}\left[\mathrm{K}^{+}\right]_{\mathrm{IN}}+\mathrm{y}\left[\mathrm{K}^{+}\right]_{\mathrm{OUT}}$, where $x$ is the intracellular space and $y$ is the extracellular space, respectively. In the perfused working rat heart, the distribution of total tissue water is $41 \%$ intracellular and $59 \%$ extracellular. ${ }^{19}$ It was assumed that $\left[\mathrm{K}^{+}\right]_{\text {Out }}$ was equal to the $\mathrm{K}^{+}$concentrations in the various cardioplegic solutions $\left(0.1,3.0,5.9,10,16\right.$, or $\left.25 \mathrm{mmol} / \mathrm{L} \mathrm{K}^{+}\right)$. Total tissue water was measured and calculated from the difference between wet and dry weight, divided by wet weight, and multiplied by $100 .{ }^{19}$

\section{Coronary Vascular Resistance}

CVR (megadyne $\cdot \mathrm{s}^{-1} \cdot \mathrm{cm}^{-5}$ ) was calculated by dividing delivery pressure $(\mathrm{mm} \mathrm{Hg})$ by flow (volume/s) from equation 2 as follows:

$$
C V R=\frac{1333 \times \mathrm{mm} \mathrm{Hg}}{(\mathrm{mL} / \mathrm{s})} \times 10^{-6}
$$

where $1 \mathrm{~mm} \mathrm{Hg}=1333$ dynes $\cdot \mathrm{cm}^{-2}$, and $10^{-6}$ is a conversion factor from dynes to megadynes. ${ }^{12}$ 